\title{
Dense-gas tracers and carbon isotopes in five $2.5<z<4$ lensed dusty star-forming galaxies from the SPT SMG sample
}

\author{
M. Béthermin ${ }^{1}$, T. R. Greve ${ }^{2}$, C. De Breuck ${ }^{3}$, J. D. Vieira ${ }^{4}$, M. Aravena ${ }^{5}$, S. C. Chapman ${ }^{6}$, Chian-Chou Chen ${ }^{3}$, \\ C. Dong $^{7}$, C. C. Hayward ${ }^{8}$, Y. Hezaveh ${ }^{9}$, D. P. Marrone ${ }^{10}$, D. Narayanan ${ }^{7,11,12}$, K. A. Phadke ${ }^{4}$, C. A. Reuter ${ }^{4}$,
} J. S. Spilker ${ }^{13}$, A. A. Stark ${ }^{14}$, M. L. Strandet ${ }^{15}$, and A. Weiß ${ }^{15}$

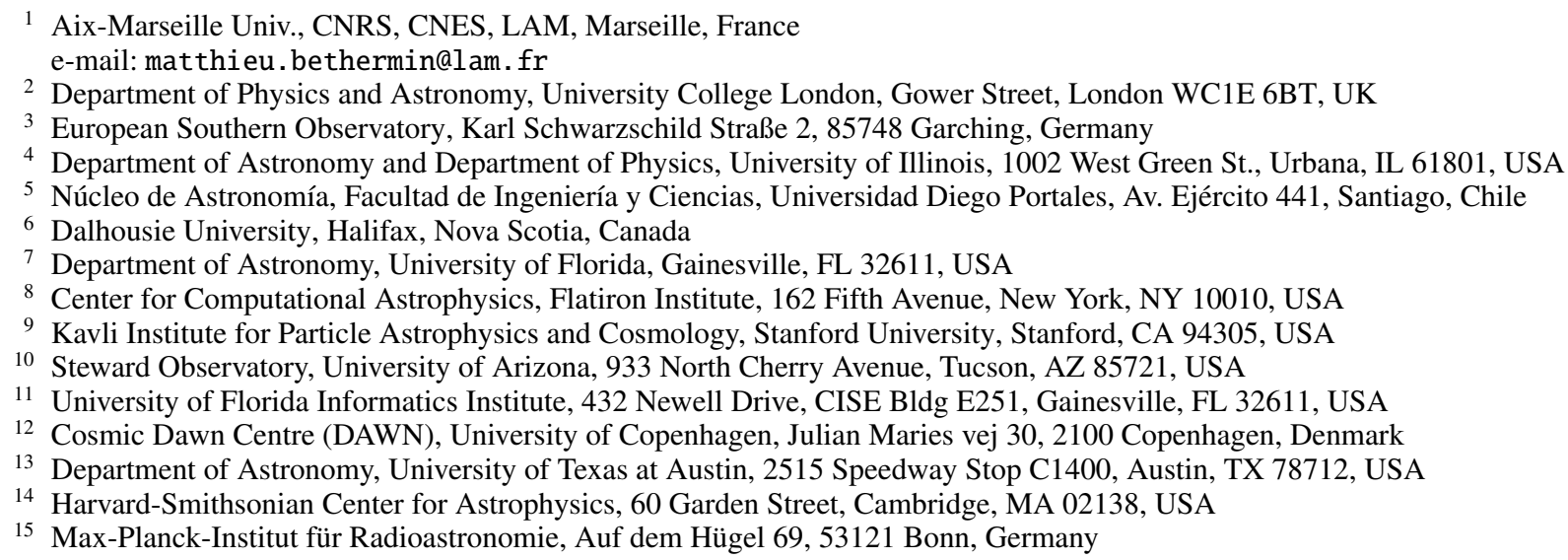

Received 23 March 2018 / Accepted 9 October 2018

\begin{abstract}
The origin of the high star formation rates (SFR) observed in high-redshift dusty star-forming galaxies is still unknown. Large fractions of dense molecular gas might provide part of the explanation, but there are few observational constraints on the amount of dense gas in high-redshift systems dominated by star formation. In this paper, we present the results of our Atacama large millimeter array (ALMA) program targeting dense-gas tracers $\left(\mathrm{HCN}(5-4), \mathrm{HCO}^{+}(5-4)\right.$, and $\left.\mathrm{HNC}(5-4)\right)$ in five strongly lensed galaxies from the South Pole Telescope (SPT) submillimeter galaxy sample. We detected two of these lines $(S / N>5)$ in SPT-125-47 at $z=2.51$ and tentatively detected all three $(S / N \sim 3)$ in SPT0551-50 at $z=3.16$. Since a significant fraction of our target lines is not detected, we developed a statistical method to derive unbiased mean properties of our sample taking into account both detections and non-detections. On average, the $\mathrm{HCN}(5-4)$ and $\mathrm{HCO}^{+}(5-4)$ luminosities of our sources are a factor of $\sim 1.7$ fainter than expected, based on the local $L_{\mathrm{HCN}(5-4)}^{\prime}-L_{\mathrm{IR}}$ relation, but this offset corresponds to only $\sim 2 \sigma$ if we consider sample variance. We find that both the $\mathrm{HCO}^{+} / \mathrm{HCN}$ and $\mathrm{HNC} / \mathrm{HCN}$ flux ratios are compatible with unity. The first ratio is expected for photo-dominated regions (PDRs) while the second is consistent with PDRs or X-ray dominated regions (XDRs) and/or mid-infrared (IR) pumping of HNC. Our sources are at the high end of the local relation between the star formation efficiency, determined using the $L_{\mathrm{IR}} /[\mathrm{CI}]$ and $L_{\mathrm{IR}} / \mathrm{CO}$ ratios, and the dense-gas fraction, estimated using the $\mathrm{HCN} /[\mathrm{CI}]$ and $\mathrm{HCN} / \mathrm{CO}$ ratios. Finally, in SPT0125-47, which has the highest signal-to-noise ratio, we found that the velocity profiles of the lines tracing dense $\left(\mathrm{HCN}, \mathrm{HCO}^{+}\right)$and lower-density $(\mathrm{CO},[\mathrm{CI}])$ molecular gas are similar. In addition to these lines, we obtained one robust and one tentative detection of ${ }^{13} \mathrm{CO}(4-3)$ and found an average $I_{1^{12} \mathrm{CO}(4-3)} / I_{13} \mathrm{CO}(4-3)$ flux ratio of $26.1_{-3.5}^{+4.5}$, indicating a young but not pristine interstellar medium. We argue that the combination of large and slightly enriched gas reservoirs and high dense-gas fractions could explain the prodigious star formation in these systems.
\end{abstract}

Key words. galaxies: ISM - galaxies: star formation - galaxies: high-redshift - galaxies: starburst - submillimeter: galaxies

\section{Introduction}

Traditionally, the molecular gas in high-redshift galaxies has been probed by observations of the rotational lines of $\mathrm{CO}$ (e.g., Solomon \& Vanden Bout 2005). Systematic CO line surveys have shown an increasing molecular gas fraction with redshift (e.g., Tacconi et al. 2010, 2013; Saintonge et al. 2013; Carilli \& Walter 2013; Dessauges-Zavadsky et al. 2015; Aravena et al. 2016; Keating et al. 2016). Other methods based on the galaxy dust content found similar results (Magdis et al. 2012; Scoville et al. 2014, 2016; Béthermin et al.
2015; Schinnerer et al. 2016). These analyses suggest that high star formation activity in massive galaxies observed at high redshift is related to their larger gas content. Furthermore, an increase in the star formation efficiency, that is, the star formation rate (SFR) relative to the total gas mass (as traced by $\mathrm{CO}$ or dust), seems necessary to account for the prodigious star formation in the most extreme systems (e.g., Engel et al. 2010; Genzel et al. 2010; Daddi et al. 2010; Magdis et al. 2011; Tan et al. 2014; Hodge et al. 2015; Silverman et al. 2015).

In contrast, we have much less information about the amount of dense, actively star-forming gas in high-redshift galaxies. The 
dense gas is usually traced using the rotational lines of highdipole molecules such as $\mathrm{HCN}$, which have $\mathrm{H}_{2}$ critical densities $\gtrsim 10^{4} \mathrm{~cm}^{-3}$ (Shirley 2015). While Kauffmann et al. (2017) argue that in some Galactic clouds the HCN(1-0) line traces gas densities similar to those traced by high- $J \mathrm{CO}$ lines $\left(10^{3} \mathrm{~cm}^{-3}\right)$, the higher $J$ transitions of $\mathrm{HCN}$ are still considered to be our best indicators of dense gas in galaxies.

As gas in the interstellar medium collapses to form stars, it passes though the density regime where the hydrogen is predominantly molecular and is dense enough to excite rotational transitions of $\mathrm{HCN}$ and $\mathrm{HCO}^{+}$into emission (Larson 1994). For stars to form, the density must be sufficiently high in the starforming cloud cores that self-gravity dominates over tidal shear. For much of the volume of a typical galaxy, the threshold density for collapse in spite of tidal shear and the threshold density for excitation of high dipole moment molecules into emission are approximately the same (Stark et al. 1989). The luminosity of the HCN line is then a good measure of the SFR. In the central kiloparsec of large galaxies, however, the density of the interstellar medium may be high enough to excite $\mathrm{HCN}$ but not high enough to resist the very much higher tidal shear in those regions of high differential rotation; the HCN line may then have a large beam-filling factor and be bright in emission from an extended, non-cloudy molecular gas, even though no star formation can take place.

In the local Universe, Gao \& Solomon (2004) found that in $\log -\log$ space, the $\mathrm{HCN}(1-0)$ luminosity is linearly proportional to SFR, as gauged by the total infrared luminosity $\left(L_{\mathrm{IR}}\right)$, with a small scatter over 2.5 decades in luminosity. The interpretation of this result is that the SFR in galaxies is primarily controlled by the dense gas fraction. Linear luminosity relations have been found using $\mathrm{CO}(3-2), \mathrm{HCO}^{+}(1-0), \mathrm{HCO}^{+}(3-2), \mathrm{HCO}^{+}$ (5-4), HCN(4-3), CS(5-4), CS(7-6), and formaldehyde dense observations of a subset of the same galaxies (Yao et al. 2003; Narayanan et al. 2005; Graciá-Carpio et al. 2006; Mangum et al. 2008, 2013; Iono et al. 2009; Juneau et al. 2009; Wang et al. 2011; Zhang et al. 2014).

Observations of Milky Way clumps in $\mathrm{HCN}(1-0)$, as well as in a variety of other dense gas tracers, by Wu et al. $(2005,2010)$ found a roughly linear relation between the SFR and the densegas mass $\left(M_{\text {dense }}\right)$ consistent with the galaxy-integrated measurements. They arrive at the same interpretation as Gao \& Solomon (2004), namely that the SFR of a galaxy scales linearly with the number of dense-gas "units" in a galaxy, with the dense-gas star formation efficiency being constant. Similarly, observations toward high visual extinction lines of sight by Lada et al. (2010, 2012) and Heiderman et al. (2010) support this interpretation. In contrast, Bigiel et al. (2016) showed that the increase in densegas fraction towards the center of M 51 leads to a decrease in the dense-gas star formation efficiency $\left(\propto L_{\mathrm{IR}} / L_{\mathrm{HCN}}^{\prime}\right)$, since there will be fewer overdense regions able to gravitationally collapse if the average density is higher.

This interpretation, however, as well as the claimed linearity of the SFR-dense gas relation is debated in the literature. For example, Bussmann et al. (2008) observe sublinear IR-HCN(3-2) luminosity relations for nearby galaxies, while García-Burillo et al. (2012) found a slightly super-linear IR-HCN(1-0) luminosity relation. Both of these sets of observations can be reconciled with simulations (e.g., Narayanan et al. 2008b, 2011). Similarly, star formation models suggest that the SFR-dense gas relationship is primarily set by the gas density probability distribution function (PDF) in galaxies. As a result, a range of SFR-dense gas mass slopes may be expected, depending on the effective density of the tracer and the exact form of the gas density PDF (e.g., Krumholz \& Thompson 2007; Narayanan et al. 2008b; Hopkins et al. 2013; Popping et al. 2014; Onus et al. 2018). In a high-pressure turbulent interstellar medium (ISM), the average gas density is expected to be higher at all physical scales with a larger fraction of the total gas mass residing at higher densities $\left(>10^{4} \mathrm{~cm}^{-3}\right)$. This type of high-pressure turbulent ISM is more likely to occur in vigorously star-forming regions than in more quiescent ISM conditions (Papadopoulos 2010). This is supported by many numerical simulations, which tend to predict a dramatic increase in the densegas fraction during major mergers causing a short ( $100 \mathrm{Myr})$ boost of the star formation efficiency (e.g., Renaud et al. 2014). However, a recent study by Fensch et al. (2017) suggests that this phenomenon could not be efficient in mergers of two gasrich galaxies at high redshift. Observational constraints are thus essential to test this result. $\mathrm{HCN}, \mathrm{HCO}^{+}$, and CS studies of local (ultra-)luminous infrared galaxies (ULIRGS) have found high gas fractions (e.g., Gao \& Solomon 2004; García-Burillo et al. 2012; Zhang et al. 2014) - but so far this has not been firmly established at high redshift.

The detections of dense-gas tracers such as $\mathrm{HCN}$ and $\mathrm{HCO}^{+}$ remain scarce at high redshift due to the faintness of these lines (typically more than 10x fainter than CO). In fact, all such detections to date were obtained with the assistance of gravitational lensing. Early efforts by, for example, Greve et al. (2006) and Gao et al. (2007) to detect HCN(1-0) in high-z starburst galaxies resulted only in upper limits. Most of the firm detections were obtained for quasars: for example, $\mathrm{HCN}(1-0)$ in the Cloverleaf at $z=2.6$ by Solomon et al. (2003), IRAS F10214+4724 at $z=2.3$ by Vanden Bout et al. (2004), and $\mathrm{J} 1409+5628$ at $z=2.6$ by Carilli et al. (2005) and $\operatorname{HCN}(5-4)$ and $\operatorname{HCN}(6-5)$ in APM08279 +5255 at $z=3.9$ (Barvainis et al. 1997; Wagg et al. 2005; Weiß et al. 2007; Riechers et al. 2010). There are even fewer published detections of star-formation-dominated objects. The HCN(3-2) line was detected in SMMJ1213511-0102 at $z=2.3$ by Danielson et al. (2011) and recently Oteo et al. (2017) reported two new detections of $\mathrm{HCN}(3-2)$ and $\mathrm{HCO}^{+}(3-2)$ in SDP.9 and SDP. 11 at $z=1.6$ and $z=1.8$, respectively (they also detected the 1-0 transition of these two molecules in SDP.9). So far, no detection at $z>2.5$ has been reported in systems dominated by star formation. However, Spilker et al. (2014) stacked all the cycle-0 Atacama large millimeter array (ALMA) spectral scans of the South Pole telescope submillimeter galaxy sample (SPT SMG, $z \sim 3.9$ ) and detected $\mathrm{HCN}(4-3), \mathrm{HCO}^{+}(4-3)$, and $\mathrm{HCO}^{+}(6-5)$.

In this paper, we present deep ALMA observations of $\mathrm{HCN}(5-4)$ in a sample of five lensed dusty star-forming galaxies (DSFGs) between $z=2.5$ and $z=4$ from the South Pole Telescope (SPT) sample (Vieira et al. 2010, 2013). We chose to target $\mathrm{HCN}(5-4)$, since the $\mathrm{HNC}(4-3)$ line is not observable beyond $z=3.2$ and we want to observe the same transition in all the sources of our sample. The transition has a typical effective excitation density of $\sim 10^{6} \mathrm{~cm}^{-3}$ (Shirley 2015 and is therefore a bone fide tracer of the densest molecular gas in galaxies. In addition to $\mathrm{HCN}(5-4)$, and since they can be observed at the same time, our observations also targeted the $\mathrm{HCO}^{+}(5-4)$ and $\mathrm{HNC}(5-4)$ lines, which are dense-gas tracers in their own right. However, these two lines are harder to interpret because of their more complex chemistry. $\mathrm{HCO}^{+}$, being an ion, is dependent on the ionization of the dense molecular clouds and is thus a less direct tracer of these high densities (Papadopoulos 2007). The line ratio between $\mathrm{HCN}$ and $\mathrm{HNC}$ is dependent on the physical conditions and varies from $\sim 1$ in dense dark clouds (Hirota et al. 1998) to $\sim 10^{-2}$ in hot and dense regions of star formation like Orion (Schilke et al. 1992). In addition to the above lines, we 
Table 1. Summary of the characteristics of our observations.

\begin{tabular}{|c|c|c|c|c|c|c|c|c|c|}
\hline Source & RA & Dec & Observing date & $t_{\mathrm{obs}}$ & $t_{\mathrm{on}}$ & PWV & Band & $N_{\text {antennae }}$ & Synthesized beam size \\
\hline SPT0103-45 & 01:03:11 & $-45: 38: 53$ & 2017-01-08 & $41 \mathrm{~min}$ & $18 \mathrm{~min}$ & $4.8 \mathrm{~mm}$ & 3 & 44 & $3.11^{\prime \prime} \times 2.92^{\prime \prime}$ \\
\hline SPT0125-47 & 01:25:07 & $-47: 23: 56$ & 2017-01-09 & $26 \min$ & $13 \min$ & $4.2 \mathrm{~mm}$ & 4 & 44 & $2.52^{\prime \prime} \times 1.96^{\prime \prime}$ \\
\hline SPT0125-50 & $01: 25: 48$ & $-50: 38: 21$ & 2016-12-29 & $60 \mathrm{~min}$ & $33 \min$ & $2.8 \mathrm{~mm}$ & 3 & 48 & $2.66^{\prime \prime} \times 2.37^{\prime \prime}$ \\
\hline SPT0300-46 & 03:00:04 & $-46: 21: 24$ & 2017-01-08 & $75 \mathrm{~min}$ & $45 \mathrm{~min}$ & $5.2 \mathrm{~mm}$ & 3 & 41 & $3.44^{\prime \prime} \times 2.72^{\prime \prime}$ \\
\hline SPT0551-50 & 05:51:39 & $-50: 58: 01$ & 2017-01-07 & $86 \min$ & $54 \mathrm{~min}$ & $5.5 \mathrm{~mm}$ & 3 & 44 & $3.22^{\prime \prime} \times 2.35^{\prime \prime}$ \\
\hline
\end{tabular}

Notes. Each source was observed in a separate ALMA scheduling block (SB). Two similar SBs were used for SPT0551-50, which required a longer integration. The total observing time and the time on source are $t_{\mathrm{obs}}$ and $t_{\mathrm{on}}$, respectively. PWV is the average precipitable water vapor level during our observations. The beam size in the table was derived using a natural weighting.

were able to simultaneously target the $J=4-3$ transition of the ${ }^{13} \mathrm{CO}$ isotopologue in four of our sources. ${ }^{13} \mathrm{C}$ is a secondary nucleus, which is not produced from fusion of hydrogen or helium in massive short-lived stars. Thus, detecting this line is a clue of previous star formation episodes in these galaxies (Hughes et al. 2008; Henkel et al. 2010).

In Sect. 2 we present our observations and our data reduction and line extraction methods. We then discuss the properties of dense-gas tracers in Sect. 3. In Sect. 4, we compare the properties of dense and lower density molecular gas tracers $(\mathrm{CO},[\mathrm{CI}])$. Finally, we present and interpret our measurements of the $\mathrm{CO}$ isotopes in Sect. 5. In this paper, we assume a Planck Collaboration XIII (2016) cosmology and a Chabrier (2003) initial mass function (IMF).

\section{Data}

\subsection{Observations}

This paper is based on ALMA cycle-4 observations (2016.1.00065.S, PI: Béthermin) of five DSFGs from the SPT sample (Vieira et al. 2013; Weiß et al. 2013; Strandet et al. 2016), which were selected for having bright apparent infrared luminosities $\left(L_{\mathrm{IR}} \geq 5 \times 10^{13} L_{\odot}\right)$ and high-quality ancillary data. ALMA bands 3 and, when needed, 4 were tuned to the redshifted frequencies of the $\mathrm{HCN}(5-4)$ transition. When possible, spare spectral windows of the correlator were placed such as to cover ${ }^{13} \mathrm{CO}(4-3), \mathrm{HCO}^{+}(5-4), \mathrm{HNC}(5-4)$, and $[\mathrm{CI}](1-0)$ (this latest line is only observable in SPT0125-47). Each spectral window covers $1.875 \mathrm{GHz}$ and contains 240 channels (coarsest frequency domain mode resolution with an online spectral averaging by a factor of 16). The previously mentioned lines are covered by two contiguous spectral windows in one of the side bands.

The sensitivities of the observations were determined to reach $5 \sigma\left(10 \sigma\right.$ for SPT0125-47) using the $L_{\mathrm{HCN}}^{\prime}-L_{\mathrm{IR}}$ of Zhang et al. (2014). Since our sources are gravitationally lensed and have an extension of $\sim 1$ arcsec (Spilker et al. 2016), we requested the most compact configuration of the array to avoid spreading the flux of our sources over several synthesized beams and thus maximizing our chances to detect the integrated emission of our objects. The characteristics of our survey are summarized in Table 1. The observations were performed on December 29th, 2016 for SPT0125-47 and between January 7th, 2017 and January 9th, 2017 for the other sources. During our observations, the precipitable water vapor level (PWV) was between 2.8 and $5.5 \mathrm{~mm}$.

\subsection{Data reduction and extraction of the spectra}

We analyzed our data with the CASA software (McMullin et al. 2007). They were initially calibrated by the standard ALMA pipeline at the observatory. In addition, we manually flagged some antennae and spectral windows with poor bandpass, phase, or amplitude calibration. In particular, the antenna DA48 had y and $\mathrm{z}$ position offsets larger than $2 \mathrm{~cm}$ in all the January 2017 observations and its data were flagged systematically. The overall quality of the rest of the data is very good.

The data were imaged using the CLEAN algorithm, and a natural weighting scheme was chosen to maximize the pointsource sensitivity. We used cleaning thresholds corresponding to $3 \sigma$ in a given channel using the map standard deviation around our source to estimate the noise. Since our lines are very broad and the signal-to-noise ratio $(\mathrm{S} / \mathrm{N})$ is low, the channels were again rebinned at the imaging stage. Because SPT0125-47 has several $5 \sigma$ detections, we only needed a rebinning by a factor of four. Similarly, since SPT0300-46 and SPT0551-50 have at least one line above $3 \sigma$, we used a factor of six. For the remaining sources, we set the rebinning factor to eight.

We decided not to subtract the continuum directly in the uv plane. Instead, the continuum was subtracted later at the line extraction stage (see Sect. 2.3). This choice was motivated by the presence of numerous broad lines in the two contiguous spectral windows of interest. The only good area of the spectrum without line contamination is between the $\mathrm{HCO}^{+}$and the $\mathrm{HNC}$ line, but is too narrow to accurately constrain the slope of the continuum (see discussion in Appendix B). The imaging of the other side band, which is free of detected lines except for SPT0125-47, showed that, at the depth of our observations, the continuum varies significantly over $2 \times 1.875 \mathrm{GHz}$. It is thus necessary to use a first-order subtraction of the continuum.

Before extracting the spectra, we checked that our sources are not significantly extended. We produced high $\mathrm{S} / \mathrm{N}$ images by combining all the channels of the four spectral windows (mode multi-frequency synthesis). We fitted a two-dimensional Gaussian model and found the width of the Gaussian to be consistent with that of the synthesized beam. Since the continuum emission of our sources is compatible with a point source at our ALMA resolution, we thus extracted their spectrum from the ALMA cube at the centroid of this Gaussian model. Using this method, we implicitly assume that the size of the regions emitting the densegas lines is not much more extended than the continuum. The extracted spectrum corresponds to the two contiguous spectral windows in the side band of $\mathrm{HCN}(5-4)$ and is presented in Fig. 1.

\subsection{Line extraction}

We extracted the lines by fitting the following model to the whole spectra. We assumed that the line profiles are Gaussian. We set a positivity prior on the line fluxes and allowed the full width at half maximum (FWHM) to vary between 200 and $1000 \mathrm{~km} \mathrm{~s}^{-1}$, which is typical for these types of sources (e.g., Aravena et al. 2016). 

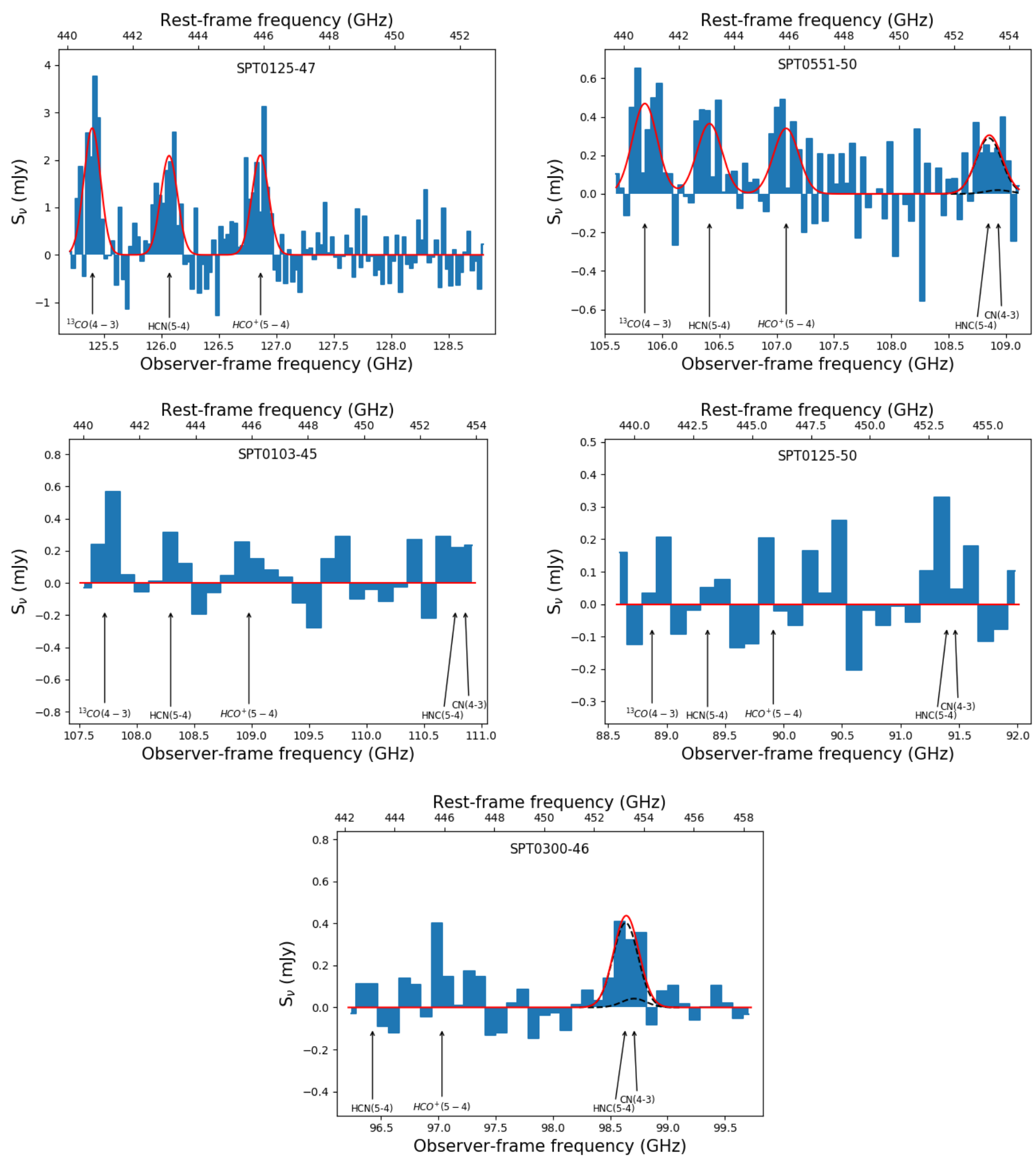

Fig. 1. Continuum-subtracted spectra of our five sources (blue filled histograms). Two spectral windows were combined to produce them. The best fit of the baseline is subtracted from the data. The red solid line is our best-fit model and the black dashed line is the best-fit decomposition of the blend between $\mathrm{HNC}(5-4)$ and $\mathrm{CN}(4-3)$. Only lines with $S / N>3$ are shown. The $\mathrm{S} / \mathrm{N}$ of the blend between $\mathrm{HNC}(5-4)$ and $\mathrm{CN}(4-3)$ in SPT0551-50 only has an S/N of 2.6 using our deblending method, which could be underestimated. However, its S/N determined in Appendix B using the moment-zero map is 3.3. We thus included the line in the plot. The three bottom panels show sources with a low $\mathrm{S} / \mathrm{N}$. Their spectra were rebinned by a factor of two in the figure to allow a better visualization of the faint lines.

We allowed a velocity offset up to $200 \mathrm{~km} \mathrm{~s}^{-1}$ compared with the expected center of the line based on the systemic redshift estimated from the band-3 spectral scan of CO. Finally, we assumed a first-order baseline for the continuum emission of the sources. All the lines $\left({ }^{13} \mathrm{CO}(4-3), \mathrm{HCN}(5-4), \mathrm{HCO}^{+}\right.$ (5-4), $\mathrm{HNC}(5-4)$, and $\mathrm{CN}(4-3))$ and the continuum baseline are fitted simultaneously. This allows us to estimate the degeneracies between the baseline parameters and the line properties. In Appendix B, we explain why this method rather than the classical extraction in moment-zero maps was preferred.

We checked a posteriori from the residuals that these are fair assumptions. We found no significant feature in the 
residual spectra. We computed the Pearson correlation coefficient between two neighboring channels and found that the correlation is lower than 0.2 for all our five residual spectra. Consequently, we can use these residuals to estimate the uncertainties on the line properties derived with our fit using a bootstrapping method. We thus took our best-fit model and added the residuals after randomly reshuffling the channels. Then, we refitted the result and saved the obtained best-fit parameters. We repeated this 10000 times. The error bars on the parameters are derived by computing the standard deviation of all these realizations. By construction, this method takes into account the degeneracies between parameters, and in particular between the baseline determination and the line fluxes. We estimated the $\mathrm{S} / \mathrm{N}$ by dividing the best-fit peak flux density by its uncertainty. Because of the combined uncertainties on the peak flux density and the width, the relative uncertainty on the line flux is slightly higher than on the peak.

Only SPT0125-47 and SPT0551-50 have sufficiently bright lines to fit them directly with reasonable constraints on the FWHM and the velocity offset for each line. For the other sources, even if there is systematically a positive signal at the position of the lines, the $\mathrm{S} / \mathrm{N}$ is often lower than 3 and the width and velocity of the lines cannot be constrained. We thus assume in our fit that these two properties are similar for all the lines. The low-frequency wing of the SPT0125-47 ${ }^{13} \mathrm{CO}(4-3)$ line is not perfectly fitted, when the width and the velocity offset of each line are independent parameters. The FWHM is smaller than for other lines: $252 \pm 45$ versus $475 \pm 128$ and $496 \pm 119$ for $\mathrm{HCN}(5-4)$ and $\mathrm{HCO}^{+}(5-4)$, respectively. However, this narrower profile is unlikely to be real (see Sect. 5.2) and might be caused by the noise. To determine the best fit of ${ }^{13} \mathrm{CO}(4-3)$, we thus performed another fit assuming that the three lines have the same width and velocity offset. The flux is then slightly higher but consistent at $1 \sigma$ with the previous value: $1.15 \pm 0.22$ versus $0.92 \pm 0.15 \mathrm{Jy} \mathrm{km} \mathrm{s}^{-1}$.

The results of our line extraction are summarized in Table 2 and the best fit is shown in Fig. 1 (red solid line). When a line is not detected at $\geq 3 \sigma$, we derived a $3 \sigma$ upper limit by summing the best-fit flux measured in the spectrum and three times the standard deviation of the flux in our multiple bootstrap realizations. Adding the signal present in the spectrum is crucial to obtain a reliable upper limit and is often forgotten in the literature. Otherwise, a source detected with an $S / N$ of 2.9999 would have an $\sim 50 \%$ probability of having a flux above the incorrectly computed $3 \sigma$ upper limit.

When HNC(5-4) and CN(4-3) are both present in the spectra, a special method is used to extract them, since they are severely blended. The deblending of these two lines is discussed in Sect. 2.6.

\subsection{Detected lines}

We detected three $>5 \sigma$ lines in SPT0125-47. ${ }^{13} \mathrm{CO}(4-3)$, $\mathrm{HCN}(5-4)$, and $\mathrm{HCO}^{+}(5-4)$ are detected at 7.2, 5.3, and 5.6 $\sigma$, respectively. In SPT0551-50, there are $\sim 3 \sigma$ peaks at the position of the four targeted lines $\left(3.4,3.0,3.1\right.$, and $2.6 \sigma$ for ${ }^{13} \mathrm{CO}$ (4-3), $\mathrm{HCN}(5-4), \mathrm{HCO}^{+}(5-4)$, and $\mathrm{HNC}(5-4)$, respectively). For the other three sources, only the blend HNC(5-4) and CN(4-3) in SPT0300-46 has an $S / N$ larger than 3 . However, these other data are very useful as lower or upper limits and allow us to derive unbiased mean properties for our sample using the method presented in Sects. 2.5 and 2.6.

Previously, $\mathrm{HCN}(5-4), \mathrm{HCO}^{+}(5-4)$, and $\mathrm{HNC}(5-4)$ have been detected at high redshift only in quasars. Danielson et al. (2011) attempted to detect them in the eyelash star-forming galaxy, but obtained only upper limits. The other transitions of these three molecules have been found in star-forming galaxies only below $z=2.5$. Our two detections in SPT0125-47 and our tentative detection in SPT0551-50 push the high- $z$ observations of these dense-gas tracers in galaxies dominated by star formation to higher redshift. When we were finishing this paper, we became aware that Chentao Yang et al. were also working on HCN detections at $z \sim 3$ in NCv1.143 and G09v1.97 and they kindly provided us with their measurements (priv. comm. ${ }^{1}$ ).

The ${ }^{13} \mathrm{CO}$ molecule has rarely been detected in star-forming galaxies at high redshift. Danielson et al. (2013) found it in SMM J2135-0102 at $z=2.3$. In addition, Weiß et al. (2013) reported two possible detections in the initial SPT SMG redshift surveys: SPT0529-54 at $z=3.36$, and SPT0532-50 at $z=3.39$. Our study doubles the number of ${ }^{13} \mathrm{CO}$ detections at high redshift.

\subsection{Unbiased average luminosities and line ratios}

Given the relatively low line detection rate of our sample, we seek sample-averaged line luminosities and ratios without being biased towards the brightest sources. To this end, we used the bootstrap analysis results presented in Sect. 2.3. In Fig. 2, we illustrate our method in the case of the average $\mathrm{HCN}(5-4)$ and $\mathrm{HCO}^{+}(5-4)$ luminosities and the flux ratio between these two lines. The probability density distribution (PDF) of the $\mathrm{HCN}(5-$ 4) (upper left panels) and $\mathrm{HCO}^{+}(5-4)$ (upper right panel) luminosities of each source are represented as colored histograms. Concerning the $>3 \sigma$ lines of SPT0125-47 and SPT0551-50, their PDFs exclude clearly the null hypothesis $\left(L^{\prime}=0\right)$ as is expected for a (tentative) detection.

However, even for non-detections, the mode of the distribution is systematically above zero. Since these PDFs are quasiGaussian, the mode is very close to the best-fit value. This is the case for the eight ${ }^{13} \mathrm{CO}(4-3), \mathrm{HCN}(5-4)$, or $\mathrm{HCO}^{+}(5-4)$ lines present in our spectral windows. The case of $\mathrm{HNC}(5-4)$ and $\mathrm{CN}(4-3)$ is not discussed here but in Sect. 2.6, since they are blended and the interpretation is more complicated. If the luminosity of these lines were strictly zero, the probability to have such a result would be $(1 / 2)^{2}=0.3 \%$, since there would be a $50 \%$ chance for each individual PDF to have a negative mode. However, their flux is unlikely to be zero and could correspond to 1$3 \sigma$, since we planned our observation to attempt detections and the lines should not be too far from the detection threshold. Negative values of the mode of the distribution would thus be rather unlikely and would request a $1-3 \sigma$ negative fluctuation of the noise in our observations. It is thus not so surprising that the modes of the PDFs of our non-detections are systematically positive. Indeed, the PDFs of such non-detections thus contain weak but potentially useful information, if we combine several objects. However, of course, these lines cannot be qualified individually as detections, since their PDFs do not exclude negative values and our measurements are thus compatible with a zero luminosity.

We determined the average luminosity of the sample using both detections and non-detections by combining their PDFs. Since our observations of each source are independent, we can assume that the measured luminosities are independent. The PDF of the sum of the luminosities of our sources can then be computed by convolving the PDFs of their individual luminosities,

1 The PhD manuscript of Yang (2017) can be downloaded at https: //tel.archives-ouvertes.fr/tel-01661478/ 
Table 2. Summary of the properties of our sources.

\begin{tabular}{|c|c|c|c|c|c|}
\hline Source & SPT0103-45 & SPT0125-47 & SPT0125-50 ${ }^{a}$ & SPT0300-46 ${ }^{a}$ & SPT0551-50 \\
\hline$z$ & 3.0917 & 2.5148 & 3.9593 & 3.5955 & 3.1641 \\
\hline Magnification $\mu^{b}$ & 14.4 & 18.9 & 26.7 & 14.0 & 6.3 \\
\hline Intrinsic $8-1000 \mu \mathrm{m}$ luminosity $L_{\mathrm{IR}}\left(10^{12} L_{\odot}\right)$ & $6.19 \pm 1.67$ & $9.63 \pm 0.90$ & $3.92 \pm 0.36$ & $5.67 \pm 1.24$ & $10.02 \pm 1.52$ \\
\hline \multicolumn{6}{|c|}{${ }^{13} \mathrm{CO}(4-3)$} \\
\hline Observed frequency $v_{\mathrm{obs}}(\mathrm{GHz})$ & 107.722 & 125.402 & 88.876 & - & 105.849 \\
\hline Velocity offset $\Delta v\left(\mathrm{~km} \mathrm{~s}^{-1}\right)$ & - & $6 \pm 19^{e}$ & - & - & $-65 \pm 92$ \\
\hline Full width at half maximum $\left(\mathrm{km} \mathrm{s}^{-1}\right)$ & - & $407 \pm 54^{e}$ & - & - & $717 \pm 235$ \\
\hline Peak flux density (mJy) & $<1.64$ & $2.67 \pm 0.37^{e}$ & $<0.47$ & - & $0.42 \pm 0.12$ \\
\hline$S / N$ & 2.7 & 7.2 & 0.1 & - & 3.4 \\
\hline Line flux $I_{13} \mathrm{CO}(4-3)\left(\mathrm{Jy} \mathrm{km} \mathrm{s}^{-1}\right)$ & $<0.73$ & $1.15 \pm 0.22$ & $<0.16$ & - & $0.32 \pm 0.15$ \\
\hline Luminosity $L^{\prime}{ }_{13} \mathrm{CO}(4-3)\left(10^{8} \mathrm{~K} \mathrm{~km} \mathrm{~s}^{-1} \mathrm{pc}^{2}\right)$ & $<15.0$ & $12.8 \pm 2.4$ & $<2.7$ & - & $15.7 \pm 7.1$ \\
\hline Average sample luminosity $L^{\prime}{ }_{13} \mathrm{CO}(4-3)^{\mathrm{c}}$ & & & $12.3 \pm 2.7$ & & \\
\hline \multicolumn{6}{|c|}{$\mathrm{HCN}(5-4)$} \\
\hline Observed frequency $v_{\text {obs }}(\mathrm{GHz})$ & 108.296 & 126.071 & 89.351 & 96.425 & 106.413 \\
\hline Velocity offset $\Delta v\left(\mathrm{~km} \mathrm{~s}^{-1}\right)$ & - & $39 \pm 45$ & - & - & $74 \pm 89$ \\
\hline Full width at half maximum $\left(\mathrm{km} \mathrm{s}^{-1}\right)$ & - & $475 \pm 128$ & - & - & $529 \pm 232$ \\
\hline Peak flux density (mJy) & $<1.28$ & $1.90 \pm 0.36$ & $<0.95$ & $<0.39$ & $0.39 \pm 0.13$ \\
\hline$S / N$ & 1.8 & 5.3 & 1.8 & 0.5 & 3.0 \\
\hline Line flux $I_{\mathrm{HCN}(5-4)}\left(\mathrm{Jy} \mathrm{km} \mathrm{s}^{-1}\right)$ & $<0.55$ & $0.96 \pm 0.23$ & $<0.27$ & $<0.32$ & $0.22 \pm 0.11$ \\
\hline Luminosity $L_{\mathrm{HCN}(5-4)}^{\prime}\left(10^{8} \mathrm{~K} \mathrm{~km} \mathrm{~s}^{-1} \mathrm{pc}^{2}\right)$ & $<11.2$ & $10.6 \pm 2.5$ & $<4.5$ & $<8.6$ & $10.6 \pm 5.1$ \\
\hline Average sample luminosity $L_{\mathrm{HCN}(5-4)^{c}}^{\prime}$ & & & $7.1 \pm 1.6$ & & \\
\hline \multicolumn{6}{|c|}{$\mathrm{HCO}^{+}(5-4)$} \\
\hline Observed frequency $v_{\mathrm{obs}}(\mathrm{GHz})$ & 108.977 & 126.864 & 89.912 & 97.031 & 107.083 \\
\hline Velocity offset $\Delta v\left(\mathrm{~km} \mathrm{~s}^{-1}\right)$ & - & $61 \pm 45$ & - & - & $170 \pm 57$ \\
\hline Full width at half maximum $\left(\mathrm{km} \mathrm{s}^{-1}\right)$ & - & $496 \pm 119$ & - & - & $318 \pm 194$ \\
\hline Peak flux density (mJy) & $<0.92$ & $1.92 \pm 0.35$ & $<0.87$ & $<0.63$ & $0.50 \pm 0.16$ \\
\hline$S / N$ & 1.1 & 5.6 & 1.5 & 1.7 & 3.1 \\
\hline Line flux $I_{\mathrm{HCO}^{+}(5-4)}\left(\mathrm{Jy} \mathrm{km} \mathrm{s}^{-1}\right)$ & $<0.37$ & $1.01 \pm 0.20$ & $<0.25$ & $<0.52$ & $0.17 \pm 0.07$ \\
\hline Luminosity $L_{\mathrm{HCO}^{+}(5-4)}^{\prime}\left(10^{8} \mathrm{~K} \mathrm{~km} \mathrm{~s}^{-1} \mathrm{pc}^{2}\right)$ & $<7.6$ & $11.1 \pm 2.2$ & $<4.0$ & $<13.8$ & $8.1 \pm 3.4$ \\
\hline Average sample luminosity $L_{\mathrm{HCO}^{+}(5-4)^{\mathrm{c}}}^{\prime}$ & & & $6.7 \pm 1.3$ & & \\
\hline \multicolumn{6}{|c|}{ HNC(5-4) and CN(4-3) (blended) } \\
\hline Obs. HNC(5-4) freque & 110.778 & - & 91.398 & 98.634 & 108.852 \\
\hline Obs. $\mathrm{CN}(4-3)$ frequency $v_{\mathrm{obs}}(\mathrm{GHz})$ & 110.860 & - & 91.466 & 98.707 & 108.933 \\
\hline Full width at half maximum $\left(\mathrm{km} \mathrm{s}^{-1}\right)$ & - & - & - & $780 \pm 212$ & $726 \pm 165$ \\
\hline$S / N$ of the blend ${ }^{d}$ & 2.3 & - & 2.5 & 3.5 & 2.6 \\
\hline Total blended flux $I_{\text {tot }}\left(\mathrm{Jy} \mathrm{km} \mathrm{s}^{-1}\right)$ & $<0.58$ & $<0.00$ & $<0.45$ & $0.37 \pm 0.11$ & $0.25 \pm 0.09$ \\
\hline Blended lum. $L_{\text {tot }}^{\prime}\left(10^{8} \mathrm{~K} \mathrm{~km} \mathrm{~s}^{-1} \mathrm{pc}^{2}\right)$ & $<11.4$ & $<0.0$ & $<7.0$ & $9.6 \pm 2.8$ & $11.5 \pm 4.4$ \\
\hline Average sample luminosity $L_{\mathrm{HNC}(5-4)^{\mathrm{c}}}^{\prime}$ & & & $4.8 \pm 1.6$ & & \\
\hline Average sample luminosity $L_{\mathrm{CN}(4-3)^{\mathrm{c}}}^{\prime}$ & & & $2.2 \pm 1.4$ & & \\
\hline
\end{tabular}

Notes. We report the properties of lines of our detections and tentative detections ( $S / N \geq 3$ ). Upper limits correspond to $3 \sigma$. The blend between HNC(5-4) and $\mathrm{CN}(4-3)$ in SPT0551-50 can be considered as a tentative detection as justified in Fig. 1 and Appendix B. ${ }^{(a)}$ We assumed the same FWHM and $\Delta v$ for all lines to fit these low S/N sources. ${ }^{(b)}$ The magnifications come from Spilker et al. (2016). We have no good lens model for SPT0551-50 and we thus assume the median magnification of the SPT sample of 6.3 (Spilker et al. 2016). ${ }^{(c)}$ The average sample luminosity is determined combining detections and non-detections using the method described in Sects. 2.5 and 2.6. ${ }^{(d)}$ The $\mathrm{S} / \mathrm{N}$ of the blend of HCN(5-4) and $\mathrm{CN}(4-3)$ is computed using the total flux of the two lines. ${ }^{(e)}$ The ${ }^{13} \mathrm{CO}(4-3)$ line of SPT0125-47 is extracted assuming that the three lines have the same width and velocity offset as discussed in Sect. 2.3.

$p\left(\sum_{k=1}^{N} L_{k}^{\prime}=N \times\left\langle L^{\prime}\right\rangle\right)=p_{1}\left(L_{1}^{\prime}\right) * p_{2}\left(L_{2}^{\prime}\right) * \ldots * p_{N}\left(L_{N}^{\prime}\right)$,

where $p_{i}\left(L_{i}^{\prime}\right)$ is the PDF of the luminosity of the $i$ th source. The average is then computed by dividing this sum by $N$, the number of sources. In practice, we do not really need to compute this convolution analytically. We can just randomly draw luminosities from our 10000 bootstrap realizations for each of our sources and then sum them. We performed this operation 10000 times to obtain the PDF of the average luminosity of the sample. In Fig. 2, the results are shown as gray filled histograms. As expected, the width of the PDF of the mean luminosity is significantly narrower than the PDFs of individual objects. We can also remark that the mean luminosity is clearly detected, since its PDF completely excludes zero.

We do not apply a positivity prior to perform our fits of the spectra. Indeed, in the hypothetical case of a line with a null flux for all sources, the bootstrap realizations would have a zero flux or a positive flux depending on the realization of the noise. The average flux would thus be positive even if the flux is zero for all sources. Practically, the absence of positivity prior would have 

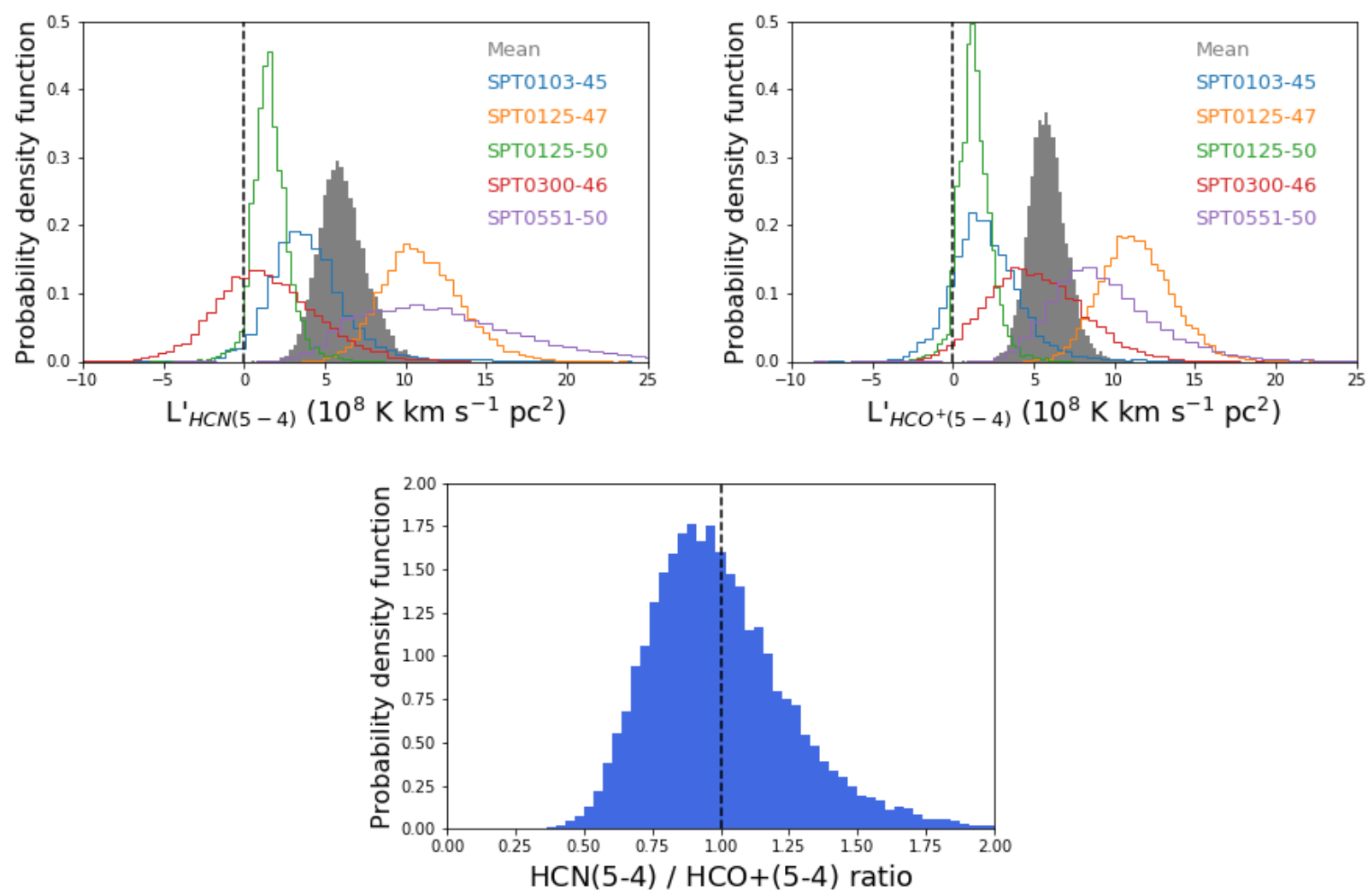

Fig. 2. Figures illustrating the method we used to derive unbiased average luminosity and line ratios (see Sect. 2.5). Upper left panel: probability density distribution of the HCN(5-4) luminosity of our sources determined using a bootstrap technique (Sect. 2.3). The gray filled histogram is the probability distribution of the mean luminosity of our five sources (see Sect. 2.5). The vertical dashed line indicates the zero flux. Upper right panel: same figure for $\mathrm{HCO}^{+}(5-4)$. Lower panel: probability density distribution of the ratio between the mean $\mathrm{HCN}(5-4)$ and the mean $\mathrm{HCO}^{+}(5-4)$ luminosities. The vertical dashed line indicate the median value of the distribution.

a minor impact, since the probability of a negative flux is small $(<10 \%)$, except for $\mathrm{HCN}(5-4)$ in SPT0300-46 and ${ }^{13} \mathrm{CO}(4-3)$ in SPT0125-50. Indeed, our unbiased mean luminosity estimates and the one derived with the positivity prior agree at better than $10 \%$, that is, $0.5 \sigma$.

This method is close to a stacking method, except that we first measure noisy fluxes and then average them instead of the contrary. However, since the lines of the various sources have different widths and the baselines are difficult to subtract reliably, we preferred this approach to a standard stacking. Indeed, the stacked lines would have had a very peculiar shape, since these are the sum of lines with various widths. It would have been impossible to use our procedure to fit the lines and the baseline simultaneously. Finally, as explained in Appendix B, flux measurements based on stacked moment maps are also unreliable, since the baseline subtraction is potentially affected by biases due to the crowded spectral windows. A stacking procedure should not be used in this case, since the systematic biases could be similar for all objects and this bias would stay roughly constant with the number of sources, while the noise would decrease in $1 / \sqrt{N}$. The measurement would thus be dominated by systematic effects and not the instrumental noise.

We used a very similar method to derive mean line flux ratios. We first computed the PDFs of the mean of the line fluxes from our bootstrap realizations using the same method as previously described. However, to avoid giving more importance to low $-z$ sources in the computation of the mean, we weighted the sources by the square of the luminosity distance. We then computed the ratio between these mean line fluxes. The PDF of the ratio between two random variables is

$p\left(r=\frac{\left\langle L_{\mathrm{A}}^{\prime}\right\rangle}{\left\langle L_{\mathrm{B}}^{\prime}\right\rangle}\right) \propto \int_{-\infty}^{+\infty}|X| p_{\mathrm{A}}(r X) p_{\mathrm{B}}(X) \mathrm{d} X$,

where $p_{\mathrm{A}}(r X)$ and $p_{\mathrm{B}}(X)$ are the PDF of the flux of the line $\mathrm{A}$ and $\mathrm{B}$ respectively, and $r$ is the line ratio in luminosity. In practice, the uncertainties on the ratio are determined by drawing realizations from the PDF of the mean luminosity of each line. The results are presented in Fig. 2. The distribution is clearly asymmetric and we thus used the 16th and 84th percentile to produce the error bars in Table 2. With this method, the contribution of the sources to the mean ratio is weighted by their luminosity. We tried to compute first the PDFs of the line flux ratios of individual sources and then average them, but this failed to get meaningful results. For low-S/N sources, the distribution is very broad with very large negative and positive outliers. Indeed, the PDF of the line flux in the denominator is compatible with zero and there are thus realizations for which the ratios are tending to $+\infty$ or $-\infty$. In Appendix C, we present the simulation used to validate this method.

\subsection{Deblending of $\mathrm{HNC}(5-4)$ and $\mathrm{CN}(4-3)$ and determination of unbiased mean ratios}

$\mathrm{HNC}(5-4)$ is blended with $\mathrm{CN}(4-3)$. To our knowledge, the only previous individual detection of this blend at high redshift was performed by Guélin et al. (2007) in the lensed quasar APM 08279+5255 (see also Riechers et al. 2007b about the detection of $\mathrm{CN}(3-2)$ in the Cloverleaf). It is also detected in the stacked spectrum of all the SPT SMG sources observed 
Table 3. Summary of the $\mathrm{CO}$ and $[\mathrm{CI}]$ data used in this paper.

\begin{tabular}{|c|c|c|c|c|c|}
\hline Source & SPT0103-45 & SPT0125-47 & SPT0125-50 & SPT0300-46 & SPT0551-50 \\
\hline$I_{12} \mathrm{CO}(4-3)\left(\mathrm{Jy} \mathrm{km} \mathrm{s}^{-1}\right)$ & $11.7 \pm 0.7$ & $23.1 \pm 0.6$ & $7.9 \pm 1.0$ & $4.9 \pm 0.5$ & $12.0 \pm 0.8$ \\
\hline$L_{12}^{\prime} \mathrm{CO}(4-3)\left(10^{10} \mathrm{~K} \mathrm{~km} \mathrm{~s}^{-1} \mathrm{pc}^{2}\right)$ & $2.22 \pm 0.13$ & $2.35 \pm 0.06^{a}$ & $1.20 \pm 0.15$ & $1.22 \pm 0.13$ & $3.39 \pm 0.24$ \\
\hline Data origin & \multicolumn{5}{|c|}{ Data from z-search programs (Weiß et al. 2013), fluxes from Bothwell et al. (2016) } \\
\hline$I_{\mathrm{CI}(1-0)}\left(\mathrm{Jy} \mathrm{km} \mathrm{s}^{-1}\right)$ & - & $6.3 \pm 0.2$ & $2.4 \pm 0.5$ & $1.8 \pm 0.8$ & - \\
\hline$L_{\mathrm{CI}(1-0)}^{\prime}\left(\right.$ intrinsic, $10^{10} \mathrm{~K} \mathrm{~km} \mathrm{~s}^{-1} \mathrm{pc}^{2}$ ) & - & $0.56 \pm 0.02$ & $0.32 \pm 0.07$ & $0.39 \pm 0.17$ & - \\
\hline Data origin & No data & This paper & \multicolumn{2}{|c|}{ z search (Bothwell et al. 2016) } & No data \\
\hline
\end{tabular}

Notes. All these data, except the new detection of [CI](1-0) in SPT0125-47, are ancillary and were extracted from the redshift-search program (Weiß et al. 2013) by Bothwell et al. (2016). ${ }^{(a)}$ Converted from the $\mathrm{CO}(3-2)$ flux using the mean flux ratio measured by Spilker et al. (2014) by stacking.

by ALMA in cycle 0 (Spilker et al. 2014). Guélin et al. (2007) found that $\mathrm{HNC}(5-4)$ is 1.74 times brighter than $\mathrm{CN}(4-3)$ by fitting simultaneously the profile of the two lines in their spectrum. This last source has a different nature than ours, but it shows that, even if $\mathrm{HNC}(5-4)$ might dominate the flux, the $\mathrm{CN}(4-3)$ contamination cannot be neglected. It is thus important to deblend the two lines in order to put a constraint on $\mathrm{HNC}(5-4)$.

To do so, we refitted the data with a slightly different method than the one described in Sect. 2.3. We fitted simultaneously all the lines in the spectrum including $\mathrm{HNC}(5-4)$ and $\mathrm{CN}(4-3)$ and forced all the lines to have the same width and velocity offset (including the sources with good $\mathrm{S} / \mathrm{N}$ ). The width and the velocity of the blended lines are thus strongly constrained by the other lines without being completely fixed. Since CN(43 ) and $\mathrm{HNC}(5-4)$ are not at the same exact frequency, these additional constraints are sufficient to extract information from the data. We also have to apply a positivity prior on the flux of $\mathrm{HNC}(5-4)$ and $\mathrm{CN}(4-3)$, since the degeneracies between the two fluxes tend to produce negative values to overfit noise patterns. Even under these assumptions, the uncertainties on the ratio for an individual source remain very high, with relative uncertainties higher than $50 \%$. We thus derived the mean luminosities of the two lines and the mean line flux ratio between $\mathrm{HNC}$ and $\mathrm{CN}$ using the method presented in Sect. 2.5. We found a mean $\mathrm{HNC}(5-4) / \mathrm{CN}(4-3)$ ratio of $1.60_{-0.82}^{+1.74}$. This value is close to the value found in the APM $08279+5255$ quasar by Guélin et al. (2007).

\section{7. $\mathrm{CO}$ and $[\mathrm{Cl}]$ lines extracted from ancillary data}

We want to compare our dense-gas tracers with lines from cold gas at lower density $(\mathrm{CO},[\mathrm{CI}])$ found by our previous redshift search programs (Weiß et al. 2013; Strandet et al. 2016). The line fluxes of the $[\mathrm{CI}](1-0)$ line and the $\mathrm{CO}$ transitions covered by the redshift-search spectral scans were extracted in Bothwell et al. (2016). For most of our sources, the $\mathrm{CO}(4-3)$ line falls in a frequency window covered by the redshift-search data. We thus chose to use this transition available for most of our sources as the reference one for $\mathrm{CO}$ in our analysis. For SPT0125-47, which is at lower redshift, only $\mathrm{CO}(3-2)$ is available. We derived the expected $\mathrm{CO}(4-3)$ flux using the line ratio measured in the stacked spectrum of the SPT SMG sources derived by (Spilker et al. 2014), $\left.I_{\mathrm{CO}(4-3)} / I_{\mathrm{CO}(3-2)}=0.7\right)$. Finally, we detected [CI](1-0) in SPT0125-47 using our new ALMA data (see Appendix A). The ancillary data used in this paper are summarized in Table 3.

\section{Dense-gas tracers: $\mathrm{HCN}, \mathrm{HCO}^{+}$, and $\mathrm{HNC}$}

\subsection{Scaling relation between the $\mathrm{HCN}$ and $\mathrm{HCO}^{+}$flux and the infrared luminosity}

The relation between the dense-gas content, traced by the HCN(1-0) line, and the SFR, traced by $L_{\mathrm{IR}}$, was found to be linear in the local Universe by Gao \& Solomon (2004). A linear correlation between $L_{\mathrm{IR}}$ and $L_{\mathrm{HCN}(1-0)}^{\prime}$ is consistent with the simple physical picture in which the giant molecular clouds (GMCs), traced by $\mathrm{HCN}$, convert a fixed fraction of their mass into stars before being disrupted (e.g., Faucher-Giguère et al. 2013; Grudić et al. 2018). However, further studies found a sublinear slope for the $J=3-2$ transition (Bussmann et al. 2008; Juneau et al. 2009), which could have an impact on the interpretation of the physics of the star formation in infrared-luminous objects and active galactic nuclei (AGN, e.g., Narayanan et al. 2008a). More recently, after considering careful aperture corrections, Zhang et al. (2014) found a linear slope in the local Universe for the $J=4-3$ transition. The $L_{\mathrm{IR}}-L_{\mathrm{CO}}^{\prime}$ relations inferred for nearby galaxies have been found to be linear for $J=6$ - 5 (Greve et al. 2014; Liu et al. 2015; Kamenetzky et al. 2016; Yang et al. 2017) and, possibly remain linear up to transitions as high as $J=12-11$ (Liu et al. 2015). Extreme galaxies, however, such as the local ULIRG population and high- $z$ starbursts, show sublinear $L_{\mathrm{IR}}-L_{\mathrm{CO}}^{\prime}$ relations for $J=7-6$ and higher, due to large amounts of energy being injected, likely via mechanical heating, into a warm, dense, and non-star-forming ISM component (Greve et al. 2014; Kamenetzky et al. 2016).

At high redshift, the $L_{\mathrm{IR}}-L_{\mathrm{HCN}}^{\prime}$ relation is poorly constrained due to the small number of detections, all of which probe the high luminosity regime. However, Riechers et al. (2007a) concluded, based on the couple of detections and upper limits obtained at that time, that the $L_{\mathrm{IR}} / L_{\mathrm{HCN}}^{\prime}$ ratio must be higher in high- $z$ starbursts and quasars. This trend agrees with the theoretical model of, for example, Krumholz \& Thompson (2007), which predicts a superlinear trend for $\mathrm{HCN}$ and $\mathrm{HCO}^{+}$at high infrared luminosity. Similarly, Narayanan et al. (2011) predicted a similar superlinear trend for $\mathrm{CO}$ at very high $L_{\mathrm{IR}}$. According to them, the median density of galaxies approaches the effective density of the molecular tracers, and the $L_{\mathrm{IR}}-L_{\mathrm{mol}}$ relation will approach the $L_{\mathrm{IR}}-M_{\text {gas }}$ relation, which in both models has a 1.5 exponent and is thus superlinear. On average, in high $L_{\mathrm{IR}}$ systems such as DSFGs, the median gas density would be much higher than in the lower luminosity systems investigated by Gao \& Solomon (2004) for which they found a linear trend.

To test if our galaxies follow the relation of Zhang et al. (2014), we converted the $L_{\mathrm{HCN}(5-4)}^{\prime}$ of our objects into $L_{\mathrm{HCN}(4-3)}^{\prime}$ 
assuming the line ratio measured in the local Universe by (Mills et al. 2013), i.e., $L_{\mathrm{HCN}(5-4)}^{\prime} / L_{\mathrm{HCN}(4-3)}^{\prime}=0.73$ ), which is also compatible with the measurements of Knudsen et al. (2007) in NGC 253. We also corrected our sources for the magnification based on Spilker et al. (2016). The results are presented in Fig. 3 (upper panel). In addition to our detections and upper limits (black squares), we also show the average $L_{\mathrm{HCN}(4-3)}^{\prime}$ and $L_{\mathrm{IR}}$ of our sample (red stars). We also compared the mean properties of our sample with the mean $L_{\mathrm{HCN}(4-3)}^{\prime}$ measured by Spilker et al. (2014) from a stacked spectrum of six SPT sources in a slightly lower redshift range than our targets. To place their data in the diagram, we divided the mean $L_{\mathrm{HCN}(4-3)}^{\prime}$ and $L_{\mathrm{IR}}$ of their six sources by their mean magnification, where a sample-median magnification of $\mu=6.3$ was assumed for sources with unknown magnification factor. Our new measurements are compatible with the previous results obtained by stacking.

All our objects have a small deficit of HCN luminosity compared to the Zhang et al. (2014) relation (black line), perhaps similar to the deficit in $\mathrm{HCN}(2-1)$ relative to infrared (IR) observed in high- $z$ quasars by Riechers et al. (2007a) (after accounting for the AGN contribution to the IR luminosity). This explains a posteriori why we did not reach our initial goal of $5 \sigma$ detections in our four $z>3$ sources (see Sect. 2.1). From the Zhang et al. (2014) relation, we expect a mean $L_{\mathrm{HCN}(5-4)}^{\prime}$ of $11.1 \times 10^{8} \mathrm{~K} \mathrm{~km} \mathrm{~s}^{-1} \mathrm{pc}^{2}$ (after converting to the 5-4 transition) based on the infrared luminosity of our sources. We measured an average flux of $(7.1 \pm 1.6) \times 10^{8} \mathrm{~K} \mathrm{~km} \mathrm{~s}^{-1} \mathrm{pc}^{2}$. This corresponds to a deficit by a factor of $1.6(0.20 \mathrm{dex})$ corresponding to a $2.5 \sigma$ difference. However, Zhang et al. (2014) used the Sanders et al. (2003) method to derive $L_{\mathrm{IR}}$, while another method was used for the SPT SMG sample (Blain et al. 2003, model 1). We estimated the median factor between the two methods by fitting the photometric points used by Zhang et al. (2014) with the SPT SMG method. We found that their method derives luminosities that are a factor of 1.27 higher. When we take this into account, the tension increases up to a factor of $2.0(0.30 \mathrm{dex})$. In contrast, the HCN(5-4) detection of NCv1.143 and the upper limit towards G09v1.97 (provided by C. Yang) are both, when converted to HCN(4-3), consistent with the observed local relation.

We checked if the deficit found with our sample could be explained by sample variance. The scatter around the Zhang et al. (2014) relation is $\sim 0.28 \mathrm{dex}$ and we thus expect a $1 \sigma$ sample variance for five objects of $0.28 / \sqrt{5}=0.125 \mathrm{dex}$. If we combine this with our measurement uncertainties, the significance of the HCN deficit has thus only a $\sim 2 \sigma$ significance. It is therefore not possible to draw any firm conclusions, especially considering the uncertainties involved in converting our $\mathrm{HCN}(5-$ 4) fluxes to $\mathrm{HCN}(4-3)$. Apart from intrinsic scatter in the 5-4/4-3 ratio, systematic effects could also be introduced by applying a locally-determined ratio to high- $z$ starburst galaxies. More data will be needed in the future.

We performed a similar analysis for the $\mathrm{HCO}^{+}$. The conversion factor from the 5-4 to the 4-3 transition based on Mills et al. (2013) is 0.64. Our results agree with the Zhang et al. (2014) ${ }^{2}$ relation (see Fig. 3, lower panel) and the average properties of our sources agree with the measurements by stacking of Spilker et al. (2014). Based on the Zhang et al. (2014) relation and the mean infrared luminosity of our sample, we expect a mean $L_{\mathrm{HCO}^{+}(5-4)}^{\prime}$ of $6.9 \times 10^{8} \mathrm{~K} \mathrm{~km} \mathrm{~s}^{-1} \mathrm{pc}^{2}$ (after applying the

\footnotetext{
2 There is a small mistake in Zhang et al. (2014) and they provided us with an updated relation (priv. comm.), which we used in our analysis: $\log \left(L_{\mathrm{IR}}\right)=1.12 \log \left(L_{\mathrm{HCO}^{+}(5-4)}^{\prime}\right)+2.83$.
}
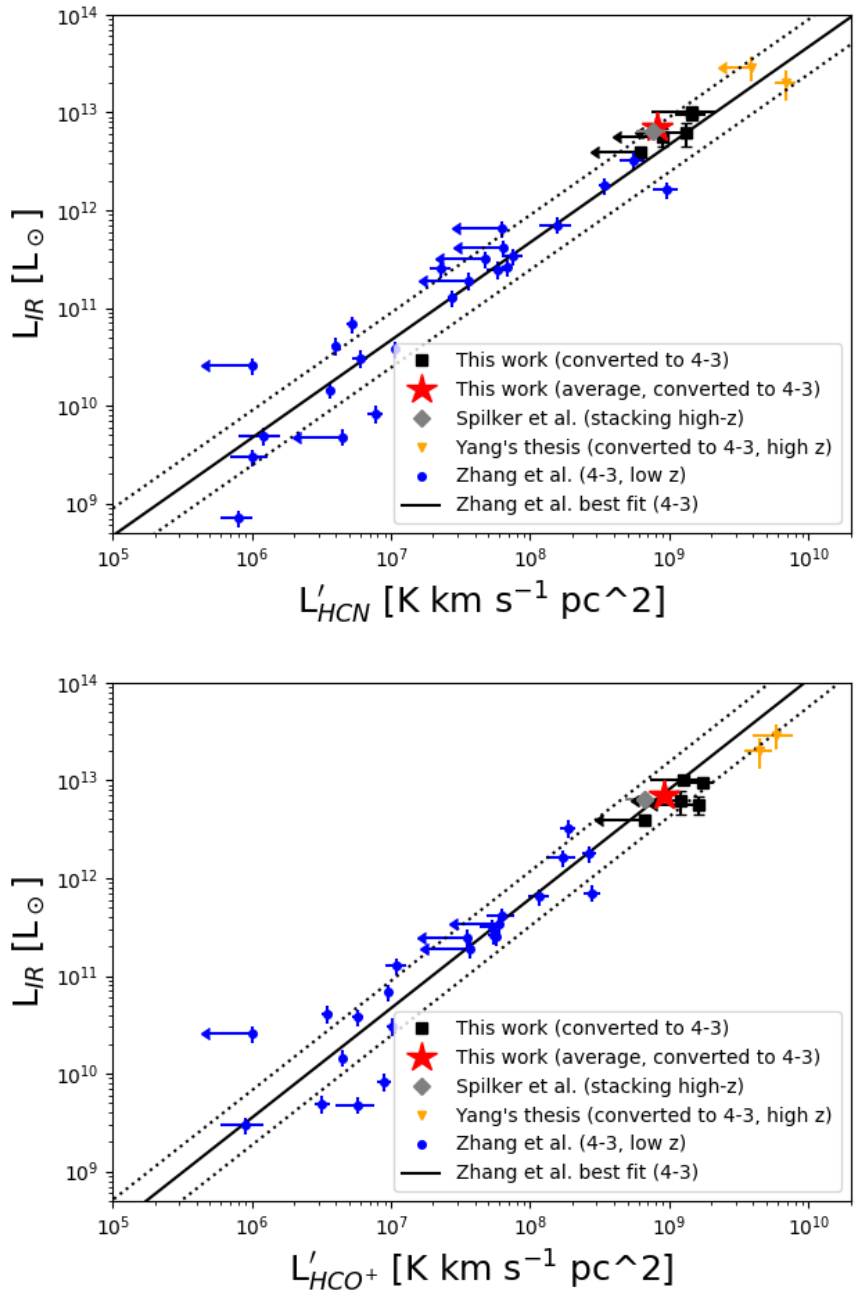

Fig. 3. Upper panel: scaling relation between the $\mathrm{HCN}(4-3)$ luminosity (converted from 5-4, see Sect. 3.1) and the total infrared luminosity. The black squares are the individual values found in our high- $z$ sample (after correcting for the lensing magnification). The red star shows the average intrinsic $L_{\mathrm{HCN}}^{\prime}$ and $L_{\mathrm{IR}}$ of our sample. The gray diamond is the mean position of the SPT SMG sample derived from the stacking of the ALMA cycle-0 data by Spilker et al. (2014). The orange downwards triangles are two H-ATLAS sources provided in Yang et al. (priv. comm.). The blue filled circles are from the local sample of Zhang et al. (2014). The black solid line is the best-fit relation of Zhang et al. (2014) and the dotted lines indicate the $1 \sigma$ intrinsic scatter around it. Lower panel: same figure but for $\mathrm{HCO}^{+}$.

correction to homogenize the $L_{\mathrm{IR}}$, see above) and we found $(6.7 \pm 1.3) \times 10^{8} \mathrm{~K} \mathrm{~km} \mathrm{~s}^{-1} \mathrm{pc}^{2}$, which is in excellent agreement. Within the scatter, the $\mathrm{HCO}^{+}$detections provided by $\mathrm{C}$. Yang are also in agreement with the relation.

\subsection{The $\mathrm{HCO}^{+}$to $\mathrm{HCN}$ flux ratio}

We compared the $\mathrm{HCO}^{+} / \mathrm{HCN} J=5-4$ flux ratios of our SPT sources (see Table 4) with $\mathrm{HCO}^{+} / \mathrm{HCN}$ ratios of low- and high- $z$ galaxies from the literature (Fig. 4). The main advantage of using flux ratios is that they cancel out the magnification factor $\mu$ if the differential magnification (Hezaveh et al. 2012; Serjeant 2012) is negligible (see Sect. 4.3). While some of the $\mathrm{HCO}^{+} / \mathrm{HCN}$ ratios from the literature were for transitions other than $J=$ $5-4$, we proceeded to compare them with our ratios under the assumption that the spectral line energy distributions (SLEDs) 
Table 4. Line flux ratios measured in our sources.

\begin{tabular}{|c|c|c|c|c|c|c|}
\hline Line flux ratio & SPT0103-45 & SPT0125-47 & SPT0125-50 & SPT0300-46 & SPT0551-50 & Mean ratio \\
\hline$I_{\mathrm{HCO}^{+}(5-4)} / I_{\mathrm{HCN}(5-4)}$ & - & $1.05_{-0.24}^{+0.30}$ & - & - & $0.78_{-0.28}^{+0.49}$ & $1.00_{-0.19}^{+0.23}$ \\
\hline$I_{\mathrm{HNC}(5-4)} / I_{\mathrm{CN}(4-3)}$ & - & $\begin{array}{r}-0.24 \\
-\end{array}$ & - & - & -0.28 & $1.60_{-0.82}^{+1.74}$ \\
\hline$I_{\mathrm{HNC}(5-4)} / I_{\mathrm{HCN}(5-4)}$ & - & - & - & - & - & $1.03_{-0.39}^{+0.59}$ \\
\hline$I_{{ }^{12}} \mathrm{CO}(4-3) / I_{13} \mathrm{CO}(4-3)$ & $>10.9$ & $19.7_{-3.1}^{+4.0}$ & $>23.1$ & - & $33.7_{-10.9}^{+21.1}$ & $26.1_{-3.5}^{+4.39}$ \\
\hline$I_{\mathrm{HCN}(5-4)} / I_{12} \mathrm{CO}(4-3)$ & $<0.067$ & $0.043_{-0.009}^{+0.010}$ & $<0.055$ & $<0.095$ & $0.020_{-0.008}^{+0.010}$ & $0.030_{-0.005}^{+0.006}$ \\
\hline$I_{\mathrm{HCN}(5-4)} / I_{[\mathrm{CI}](1-0)}$ & - & $0.158_{-0.032}^{+0.038}$ & $<0.215$ & $<1.374$ & $\begin{array}{r}-0.008 \\
-\end{array}$ & $0.129_{-0.026}^{+0.031}$ \\
\hline
\end{tabular}

Notes. The mean ratio is computed combining all the sources for which these two lines were observed (see the description of the method in Sects. 2.5 and 2.6). For the ratios between two dense-gas tracers, we provided values only when the two lines are detected at more than $2 \sigma$. Otherwise, the ratio is often compatible at $2 \sigma$ with both zero and infinity. Concerning the ${ }^{12} \mathrm{CO} /{ }^{13} \mathrm{CO}$, only SPT0125-50 is observed and not detected and we consequently derived a lower limit on this ratio, since ${ }^{12} \mathrm{CO}$ is well detected. For the ratio between $\mathrm{HCN}(5-4)$ and $\mathrm{CO}(4-3)$ or $[\mathrm{CI}](1-0)$, we derived upper limits, since the denominator $(\mathrm{CO}$ or $[\mathrm{CI}])$ is often detected.

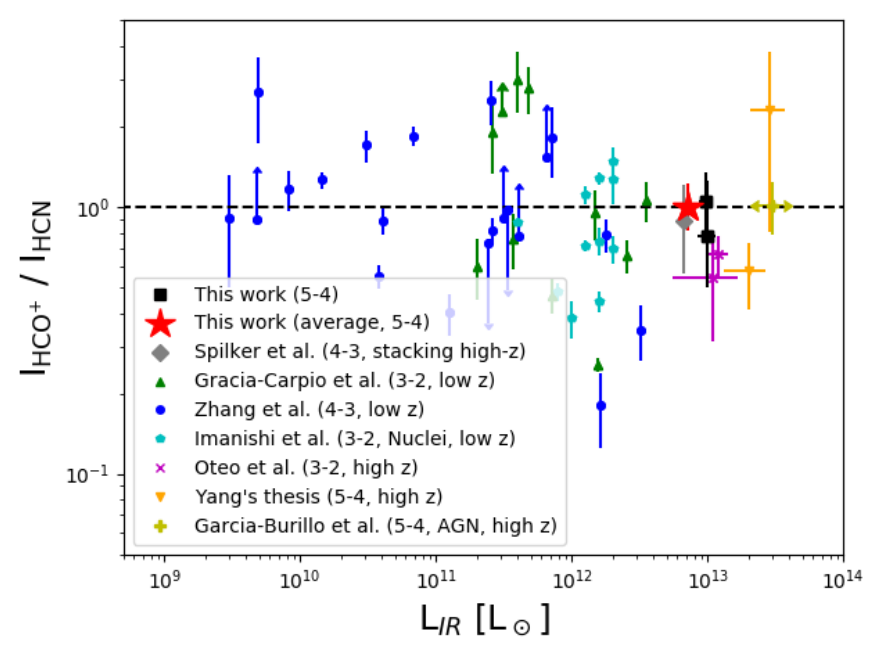

Fig. 4. Line flux ratio between $\mathrm{HCO}^{+}$and $\mathrm{HCN}$ as a function of the infrared luminosity. The black squares are the individual values obtained for our sources (only sources with two $>3 \sigma$ lines are shown) and the red star is the average value of our five objects. The gray diamond is the mean ratio found by Spilker et al. (2014) using a stacking of the cycle-0 SPT SMG spectra. The green triangles are the ratios of the 3-2 transitions in the local sample of Graciá-Carpio et al. (2008) and blue filled circles are the 4-3 ratios in the low- $z$ sample of Zhang et al. (2014). The cyan pentagons represent the 3-2 ratios measured in local active nuclei by Imanishi et al. (2016). The purple crosses shows the 3-2 ratio measured in $z \sim 1.5$ lensed star-forming galaxies by Oteo et al. (2016). The orange downwards-facing triangles are two H-ATLAS sources provided by Yang et al. (priv. comm.). The yellow plus is the 5-4 flux ratio found by García-Burillo et al. (2006) in the APM08279+5255 quasar (see also Riechers et al. 2010 for the 6-5 transition).

of the $\mathrm{HCO}^{+}$and $\mathrm{HCN}$ are similar. In addition to the average value $\left(1.00_{-0.19}^{+0.23}\right)$ of our sample, we put in our diagram only the sources that have at least a $3 \sigma$ signal at the position of each line (SPT0125-47 and SPT0551-50). For the other sources with $<3 \sigma$ signal, both the numerator $\left(\mathrm{HCO}^{+}\right.$flux $)$and the denominator (HCN flux) are compatible with zero at $3 \sigma$ (see Sect. 2.5). The PDF of their ratio is thus compatible with both zero and infinity at $3 \sigma$ and deriving upper or lower limits does not make sense. Nevertheless, we checked the average line ratio derived for these three other sources and found that it is compatible at $1 \sigma$ with the average value derived for the fives sources and the two individual measurements, but with very large uncertainties $\left(0.98_{-0.40}^{+0.73}\right)$.
Similar to the local star-forming samples of Graciá-Carpio et al. (2008) and Zhang et al. (2014), the mean ratio of our sample is compatible with unity. This is consistent with the ratio derived for the $J=4-3$ transitions using the stacked spectra of Spilker et al. (2014), but with improved uncertainties. The mean ratio of our sample is $2 \sigma$ higher than in the two high-z star-forming galaxies of Oteo et al. (2016). NCv1.143 (Yang et al.) has similar values to these two objects, but G09v1.97 has a much higher value ( 2). However, all these high- $z$ sources are in the intrinsic scatter of the local relation. As in the low $z$ Universe, the ratio varies significantly within the population of lensed DSFGs. Braine et al. (2017) found that low-metallicity regions of local galaxies $\left(<0.5 Z_{\odot}\right)$ have a high $\mathrm{HCO}^{+} / \mathrm{HCN}$ flux ratio $(\sim 2)$ instead of a ratio close to unity. Finding a unity ratio in our sources could be consistent with an already mature ISM at early cosmic times, but a larger statistical sample will be necessary to confirm or not this possibility.

Recently, Imanishi et al. (2016) and Izumi et al. (2016) found a $\mathrm{HCO}^{+} / \mathrm{HCN}$ flux ratio $(\sim 0.5)$ for both the $J=3-2$ and $J=4-3$ transitions that is lower in local AGNs than in star-forming galaxies ${ }^{3}$ and proposed that this quantity could be used to identify AGNs. Izumi et al. (2016) discussed two explanations for the low $\mathrm{HCO}^{+} / \mathrm{HCN}$ : an enhanced abundance of $\mathrm{HCN}$ compared with $\mathrm{HCO}^{+}$coming from the complex chemical and radiative mechanisms involving these molecules in the neighborhood of an AGN or a systematically higher gas density around AGNs. We found a ratio close to unity, which is consistent with our objects being star-formation dominated. However, we should interpret this simple diagnostic with caution, since some AGNs of Imanishi et al. (2016) and the APM08279+5255 quasar at $z=3.9$ have flux ratios close to unity.

\subsection{The HNC to HCN flux ratio}

Other important diagnostics can be performed using the isomer ratio between $\mathrm{HNC}$ and $\mathrm{HCN}$. HNC traces gas of similar density to $\mathrm{HCN}$, but the observed $\mathrm{HNC} / \mathrm{HCN}$ flux ratio is close to unity in dark clouds and up to 100 times smaller in hot environments (Schilke et al. 1992; Hirota et al. 1998). In addition, Aalto et al. (2007) showed that the HNC/HCN flux ratio can be above unity in local starbursts, which is not intuitive because HNC should be more easily destroyed than $\mathrm{HCN}$ by the strong radiation fields and high temperatures in these objects. They proposed

\footnotetext{
3 In the original articles, the authors used the $\mathrm{HCN} / \mathrm{HCO}^{+}$flux ratio (the inverse) and thus discussed a high ratio instead of a low ratio in this paper.
} 


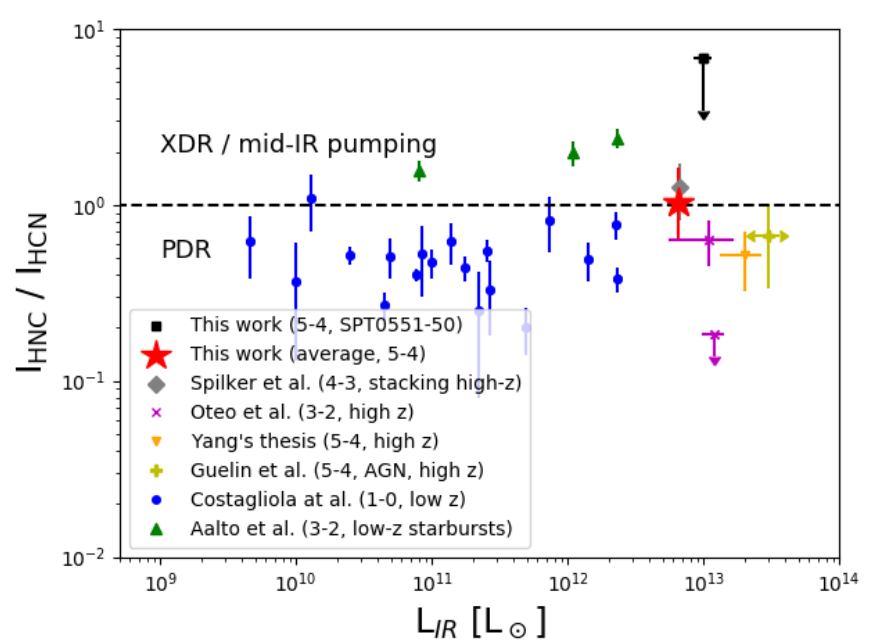

Fig. 5. Line flux ratio between $\mathrm{HNC}$ and $\mathrm{HCN}$ as a function of the infrared luminosity. The red star is the mean ratio found in our sources and the gray diamond is the mean ratio found by Spilker et al. (2014) using a stacking of the cycle-0 SPT SMG spectra. The black square is the upper limit determined for SPT0551-50. For the other sources, the individual ratio cannot be constrained, since both lines are too weak to derive an upper or a lower limit. The blue filled circles represent the local sample of (Costagliola et al. 2011, 1-0 transition). The green triangles are the 3-2 transitions in the Arp220, Mrk231, and NGC4418 starbursts (Aalto et al. 2007). The purple crosses are the two $z \sim 1.5$ lensed star-forming galaxies of Oteo et al. (2016) and the yellow plus is the APM08279+5255 quasar (Guélin et al. 2007, see also Riechers et al. 2010 for the 6-5 transition). The orange downwards triangles are two H-ATLAS sources provided in Yang's Thesis (priv. comm.).

two possible explanations: HNC is excited by mid-IR pumping of its rotational levels or X-ray dissociation regions (XDRs) have an impact on the abundance of HNC. Since then, the case of Arp220 has been extensively investigated and more signs of $\mathrm{HCN}$ or HNC pumping have been identified (e.g., Aalto et al. 2015; Galametz et al. 2016). Finally, a similar scenario was also discussed in Weiß et al. (2007) to explain HCN luminosity in the high-redshift quasar APM08279+5255.

Because of the blending of $\mathrm{HNC}(5-4)$ with $\mathrm{CN}(4-3)$, we derived only a mean $\mathrm{HNC} / \mathrm{HCN}$ ratio using the method described in Sect. 2.6, and found a ratio compatible with unity $\left(1.03_{-0.39}^{+0.59}\right)$. This ratio is compatible with the one obtained by stacking of the HNC(4-3) and HCN(4-3) lines by (Spilker et al. 2014, gray diamond in Fig. 5). Since the $\operatorname{HCN}(4-3)$ line is not blended, it is thus reassuring to find similar values. Our sources are at the border between the regime dominated by photodissociation regions (PDRs) and the domain, where XDR and/or mid-IR pumping are necessary to explain the line ratios. In Fig. 5, we compare the mean ratio found for our sample (derived using the method described in Sect. 2.6) with local and distant samples. The mean $\mathrm{HNC} / \mathrm{HCN}$ ratio of our sample is a factor of approximately three above the local IRAM/30 m sample of Costagliola et al. (2011), but lower by a factor of approximately two than the starbursts of Aalto et al. (2007). Our average measurements are only $1 \sigma$ above the measurements of APM08279+5255 by Guélin et al. (2007), SDP.9 of Oteo et al. (2017), and NCv1.143 in Yang's Thesis, but an order of magnitude above the upper limit for SDP.11 (Oteo et al. 2017).

\section{Dense gas versus lower density tracers (CO, $[\mathrm{Cl}])$}

The origin of the strong star formation in the most extreme high-redshift starbursts is a source of intense debates (e.g.,
Engel et al. 2010; Daddi et al. 2010; Hayward et al. 2011, 2013; Carilli \& Walter 2013; Casey et al. 2014; Narayanan et al. 2015). Large gas reservoirs are not sufficient to explain the SFR of the most extreme systems and a temporary increase of the star formation efficiency, measured relative to the total gas mass as traced by $\mathrm{CO}(1-0)$ or $\mathrm{CO}(2-1)$, is necessary (e.g., Daddi et al. 2010; Genzel et al. 2010; Sargent et al. 2014). Gao et al. (2007) and Daddi et al. (2010) suggested that the increase of the star formation efficiency in these objects is linked to an increase of the dense-gas fraction (DGF). The dense gas fraction is thus one of the keys to understanding the nature of this type of sources. We note, however, that an increase in the dense-gas star formation efficiency is not required in this picture.

\subsection{Dense gas fraction versus infrared luminosity}

In this section we compare the flux ratio between $\operatorname{HCN}(5-4)$, which probes the dense gas, and [CI](1-0), which is thought to be a tracer of the bulk gas reservoir. For the three sources in our sample with $[\mathrm{CI}](1-0)$ measurements, we therefore adopt this ratio as a proxy for the DGF.

To build a reference sample in the local Universe, we combined the HCN(4-3) sample of Zhang et al. (2014) with the [CI] fluxes from the local Herschel/spectral and photometric imaging receiver (SPIRE) spectroscopic sample of Rosenberg et al. (2015). We adopted the aperture-corrected integrated [CI] fluxes published by Rosenberg et al. (2015), and, similarly, the aperture-corrected integrated $\operatorname{HCN}(4-3)$ fluxes published by Zhang et al. (2014). Finally, the HCN(4-3) fluxes were converted into $\mathrm{HCN}(5-4)$ using the conversion described in Sect. 3.1.

In the left panel of Fig. 6, we show the ratio between $\mathrm{HCN}$ (5-4) and [CI](1-0) versus $L_{\mathrm{IR}}$ for our three sources observed in $[\mathrm{CI}](1-0)$. Also shown is their mean flux ratio, as well as the upper limit derived from the stacked spectra of SPT sources (Spilker et al. 2014). Our sources are seen to be consistent with the stacked SPT value, but at the limit of the upper envelope of the local reference sample.

Given the lack of low- $J$ CO transitions for some of our sources, we are not able to gauge the dense gas fraction using $\mathrm{HCN}($ mid- $J$ )/CO(low- $J$ ) ratio. Instead we opted for the $J=4-3$ $\mathrm{CO}$ transition, since it is available for most of our sources, and examine the $\mathrm{HCN}(4-3) / \mathrm{CO}(4-3)$ ratio. $\mathrm{HCN}(4-3)$ and $\mathrm{CO}(4-3)$ have similar upper level energies $\left(E_{\mathrm{J}} / k_{\mathrm{B}} \sim 40-55 \mathrm{~K}\right)$ and both trace dense gas, albeit $\mathrm{HCN}(4-3)$ has a $\sim 400 \times$ higher critical density than $\mathrm{CO}(4-3)\left(\sim 8 \times 10^{6} \mathrm{~cm}^{-3}\right.$ vs. $\left.\sim 10^{4} \mathrm{~cm}^{-3}\right)$. For the local reference sample we again adopt Zhang et al. (2014), and use their directly measured $\mathrm{HCN}(4-3)$ fluxes and the $\mathrm{CO}$ (4-3) fluxes from Rosenberg et al. (2015) to form the HCN(4$3) / \mathrm{CO}(4-3)$ ratios. In the right panel of Fig. 6, we compare the $\mathrm{HCN}(4-3) / \mathrm{CO}(4-3)$ flux ratios of our sources, where we have converted $\mathrm{HCN}(5-4)$ to $\mathrm{HCN}(4-3)$. There are not a lot of $\mathrm{HCN}(4-3) / \mathrm{CO}(4-3)$ measurements of high- $z$ sources available in the literature, and we therefore extended our comparison to $\mathrm{HCN}(5-4) / \mathrm{CO}(5-4)$ and $\mathrm{HCN}(3-2) / \mathrm{CO}(3-2)$ in order to facilitate a comparison with other high- $z$ starbursts. Our mean $\mathrm{HCN}(4-3) / \mathrm{CO}(4-3)$ ratio is in the scatter of the local values and compatible with the upper limit on G09v1.97 (Yang's Thesis). In contrast, our sources are on average a factor of 1.6 lower than the stacking measurement of Spilker et al. (2014) on the full cycle-0 SPT SMG sample $(\sim 2 \sigma$ difference) and a factor of approximately three below the high- $z$ measurements of Oteo et al. (2016) in SDP.9, Danielson et al. (2011) in SMM J2135-0102, and NCv1.143 in Yang's Thesis. 

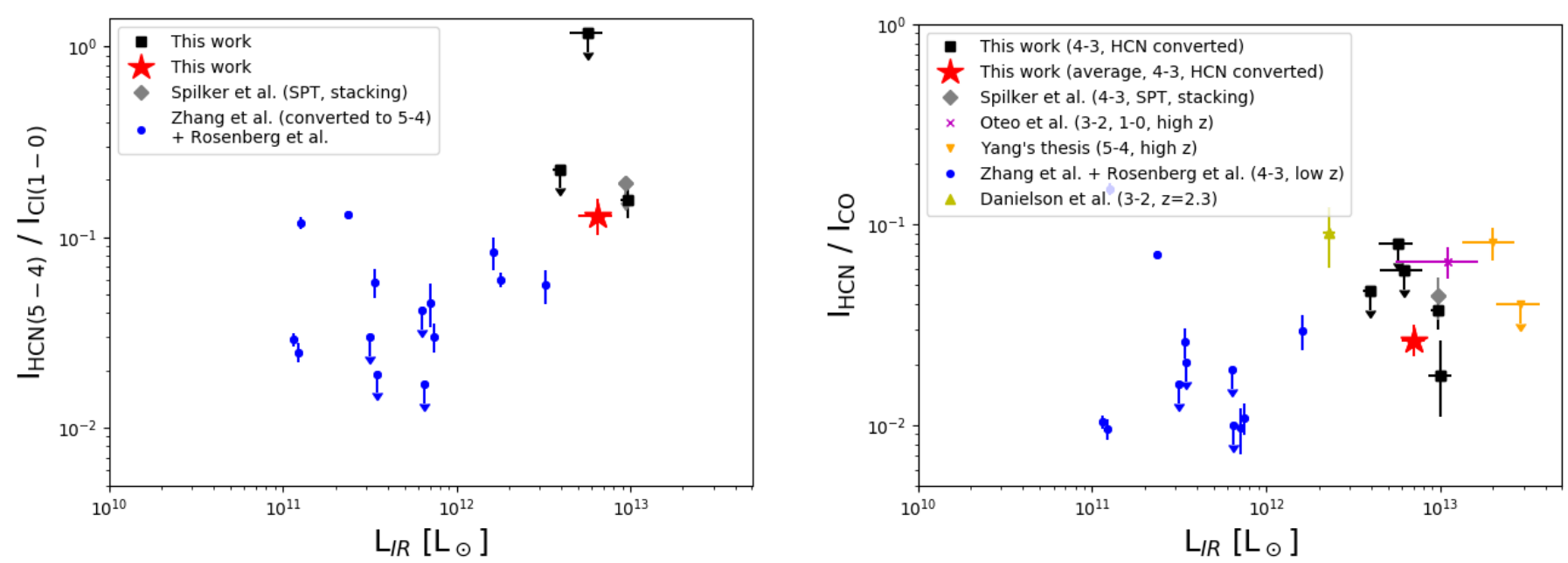

Fig. 6. Left panel: flux ratio between $\mathrm{HCN}(5-4)$ and $[\mathrm{CI}](1-0)$ as a function of the infrared luminosity. Our sources are represented by black filled squares and the average by a red star. The gray diamond represents the stacking of the cycle-0 SPT SMG spectra (Spilker et al. 2014). The blue filled circles are the local HCN(4-3) measurements of Zhang et al. (2014), where HCN(4-3) is converted into HCN(5-4) following the recipe described in Sect. 3.1, combined with the [CI](1-0) measurements of Rosenberg et al. (2015). Right panel: ratio between HCN(4-3) and ${ }^{12} \mathrm{CO}(4-3)$ as a function of the infrared luminosity. As explained in Sect. 4.1, we used the $\mathrm{HCN}(5-4) / \mathrm{CO}(5-4)$ or $\mathrm{HCN}(3-2) / \mathrm{CO}(3-2)$ ratios, when the 4-3 transitions were not available. The purple cross is the measurement of Oteo et al. (2016) in SDP.9. The orange downwards triangles are two H-ATLAS sources provided in Yang's Thesis (priv. comm.). The yellow triangle is the measurements of Danielson et al. (2011) in SMM J2135-0102.

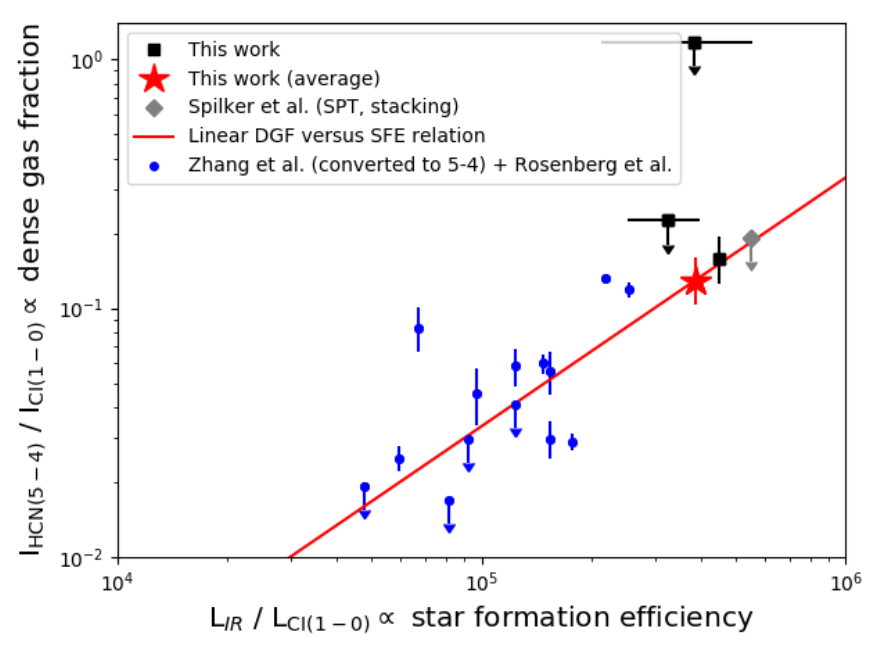

Fig. 7. Ratio between $\mathrm{HCN}(5-4)$ and $[\mathrm{CI}](1-0)$, tracing the dense-gas fraction, as a function of the ratio between the infrared and the $[\mathrm{CI}](1-$ $0)$ luminosity, tracing the star formation efficiency. The data comes from the same references as in Fig. 6. The red solid line is a linear relation between the dense-gas fraction and the star formation efficiency (DGF $\propto \mathrm{SFE}$ ), which normalization has been set to match the mean value of our sample (red star).

\subsection{A link between dense-gas fraction and star formation efficiency}

Gao et al. (2007) found a correlation between $L_{\mathrm{HCN}(1-0)}^{\prime} / L_{\mathrm{CO}(1-0)}^{\prime}$ and $L_{\mathrm{IR}} / L_{\mathrm{CO}(1-0)}^{\prime}$, which they interpreted as a correlation between the DGF and the SFE (with respect to the total gas mass). In Fig. 7, we performed a similar diagnostic using [CI](1-0) instead of $\mathrm{CO}(1-0)$ and $\mathrm{HCN}(5-4)$ instead of $\mathrm{HCN}(1-0)$. Our SPT sources are consistent with the trend of DGF versus SFE found in our local reference sample described in Sect. 4.1. A similar result is found if we use $\mathrm{CO}(4-3)$ instead of $[\mathrm{CI}](1-0)$ (see Fig. D.1). This seems to indicate that the correlation between the DGF and the SFE is still valid for the most star-forming systems at high redshift and for the densest gas probed by the high- $J$ transitions of HCN. However, an artificial correlation can appear in diagrams representing $\mathrm{A} / \mathrm{C}$ versus $\mathrm{B} / \mathrm{C}$. In Appendix D, we confirm that it is not the case for our analysis using two different total gas mass tracers in the $x$ and $y$ axis.

The relation linking our local reference sample and the SPT sources suggests that DGF $\propto$ SFE (solid red line in Fig. 7). This result agrees with the suggestion by Gao et al. (2007) and Daddi et al. (2010) that the high SFEs in starbursts are directly connected to their DGF. The link between these two quantities is not surprising, if, as suggested by the various physical models cited previously, the amount of dense gas, rather than the total gas reservoir, drives the SFR. Unfortunately, our observations do not allow us to form a conclusion about the mechanism that causes these high DGFs. The sources in our sample are at the high end of the SFE and DGF distribution of our local reference sample. Their impressive SFRs are thus caused by a combination of large gas reservoirs (Aravena et al. 2016) coupled with a high DGF.

\subsection{Similarity of the line profiles in SPT0125-47 and differential lensing}

In SPT0125-47, dense-gas tracers are detected at more than $5 \sigma$ and it is thus possible to compare the line profile of $\mathrm{HCN}$ and $\mathrm{HCO}^{+}$with $[\mathrm{CI}]$ and $\mathrm{CO}$ (see Fig. 8). Our high-S/N [CI] detection and the ancillary $\mathrm{CO}(3-2)$ line detection from the redshift search have a similar asymmetric profile with a much broader redshifted tail. A similar asymmetry is found for $\mathrm{HCN}(5-4)$ and $\mathrm{HCO}^{+}(5-4)$. This suggests that molecular regions from the lowest to the highest density are distributed in the same way across this object.

The similarity of the profiles also suggests that differential lensing (Hezaveh et al. 2012; Serjeant 2012) should not be too strong in this object. Concerning the other objects of our sample, we do not have a sufficient $\mathrm{S} / \mathrm{N}$ to be able to check the similarity of the profiles between the ${ }^{12} \mathrm{CO}$ lines and the dense-gas lines. However, as discussed in Gullberg et al. (2015), most of 


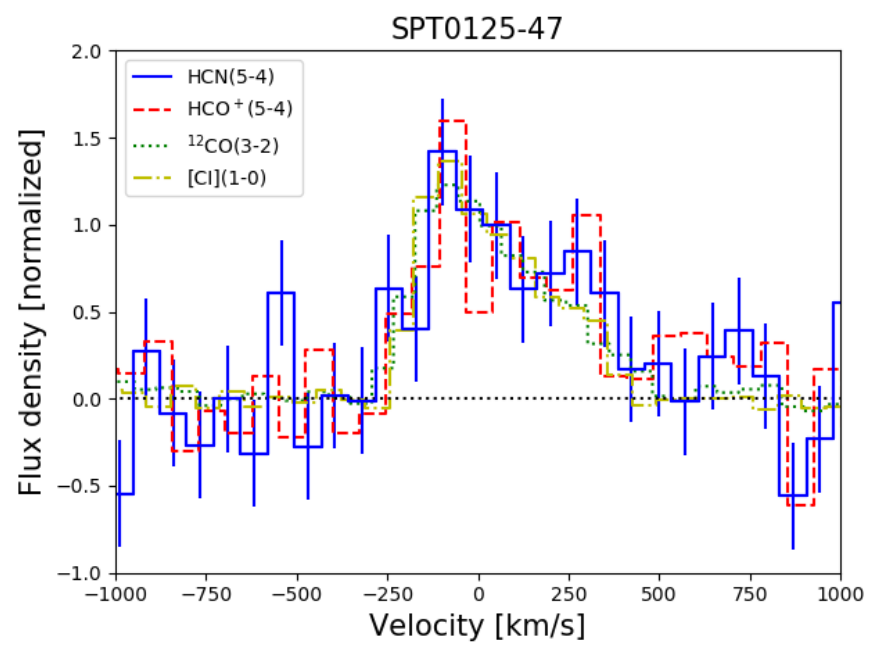

Fig. 8. Comparison of the velocity profile of the dense-gas lines $\left(\mathrm{HCN}(5-4)\right.$ in blue solid line and $\mathrm{HCO}^{+}(5-4)$ in red dashed line, and other gas tracers $(\mathrm{CO}(3-2)$ in green dotted line and $[\mathrm{CI}](1-0)$ in yellow dot-dash line. We plotted the channel RMS uncertainties of $\mathrm{HCN}(5-4)$ $\mathrm{HCO}^{+}(5-4)$ has similar channel uncertainties (not plotted). ${ }^{12} \mathrm{CO}$ and $[\mathrm{CI}]$ have a much better $\mathrm{S} / \mathrm{N}$ and their uncertainties can be neglected. We normalized all the lines to have $\int S_{\nu} \mathrm{d} v=500 \mathrm{Jy} \mathrm{km} \mathrm{s}^{-1}$. With this normalization, the peak flux of a rectangular line with a typical $500 \mathrm{~km} \mathrm{~s}^{-1}$ width is unity.

the SPT sources have for instance similar $\mathrm{CO}$ and [CII] profiles. Except if the dense-gas lines have a fundamentally different spatial distribution than lower density tracers, we have no good reason to expect a priori any strong differential lensing effect. We thus neglected this effect in this paper. The only way to measure the impact of the differential lensing on integrated fluxes would be to perform high-resolution imaging of dense-gas lines, but it is out of reach of ALMA in a reasonable amount of time.

\section{Properties of the $\mathbf{C O}$ isotopic lines}

\subsection{Flux ratios between ${ }^{13} \mathrm{CO}$ and ${ }^{12} \mathrm{CO}$}

As explained in the Introduction, the $\left[{ }^{12} \mathrm{C}\right] /\left[{ }^{13} \mathrm{C}\right]$ abundance ratio has been proposed as a diagnostic of the evolutionary stage of a galaxy, since different nuclear reactions produce ${ }^{12} \mathrm{C}$ and ${ }^{13} \mathrm{C}$; the former is produced via triple alpha nuclear processes in young massive stars, while the latter is produced in the CNO cycle in evolved asymptotic giant branch (AGB) stars (Wilson \& Rood 1994).

A high $\left[{ }^{12} \mathrm{C}\right] /\left[{ }^{13} \mathrm{C}\right]$ abundance ratio, therefore, could indicate a chemically young and largely unprocessed ISM (Hughes et al. 2008; Henkel et al. 2010). However, other physical mechanisms could result in a high $\left[{ }^{12} \mathrm{C}\right] /\left[{ }^{13} \mathrm{C}\right]$ ratio, namely star formation from a top-heavy IMF in young starbursts (e.g., Romano et al. 2017; Zhang et al. 2018) - with the latter being the result of extreme cosmic-ray-dominated, star formation regions rather than a metal-poor gas. In intense starburst environments, the cosmic ray heating may be so severe that dense, deeply embedded molecular cores are significantly heated, resulting in a raise of the Jeans mass floor (Papadopoulos et al. 2011).

The $\left[{ }^{12} \mathrm{C}\right] /\left[{ }^{13} \mathrm{C}\right]$ ratio is typically constrained indirectly from observations of $\mathrm{CO}, \mathrm{HCN}$, and $\mathrm{HCO}^{+}$and their ${ }^{13} \mathrm{C}$ isotopologues. This approach, however, is complicated by isotope fractionation where chemical reactions involving ${ }^{13} \mathrm{C}$ are energetically favored over the same reactions involving ${ }^{12} \mathrm{C}$ (Watson et al. 1976; Langer et al. 1984). The expectation from

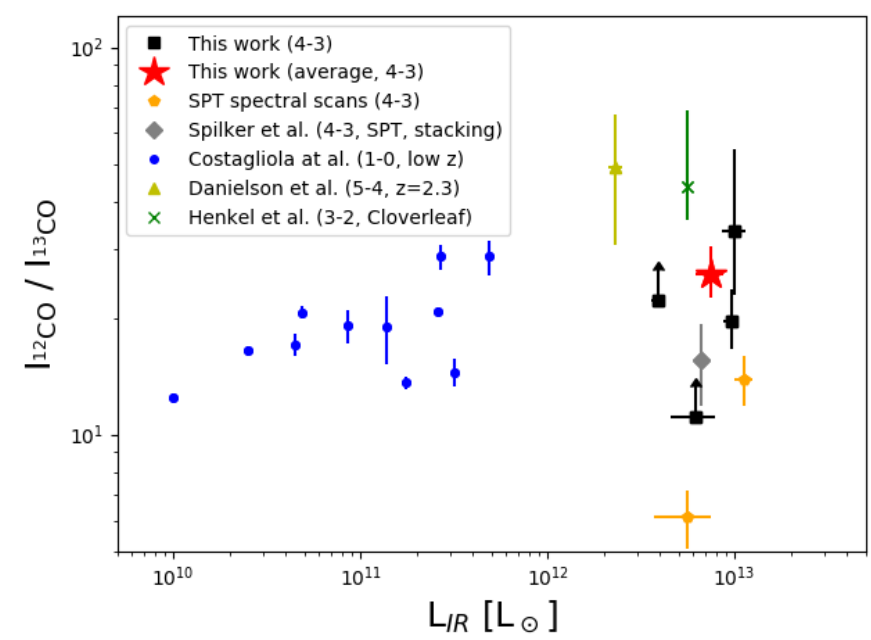

Fig. 9. Flux ratio between the ${ }^{13} \mathrm{CO}$ and ${ }^{12} \mathrm{CO}$ as a function of the infrared luminosity. The same transition of both lines is used to compute these ratios. Our sources are represented by black filled squares and their average properties by a red star. The two serendipitous detections obtained during the first redshift-search campaign in SPT0529-54 and SPT0532-50 (Weiß et al. 2013) are shown using orange pentagons. The gray diamond shows the average ratio measured by Spilker et al. (2014) using a stacking of the full redshift-search SPT sample. The local sample from Costagliola et al. (2011) is plotted with a blue filled circle. The yellow triangle and the green cross are the high- $z$ measurements of Danielson et al. (2013) in SMM J2135-0102 and Henkel et al. (2010) in the Cloverleaf quasar.

isotope fractionation is that $\left[{ }^{12} \mathrm{CO}\right] /\left[{ }^{13} \mathrm{CO}\right]$ provides a lower limit to $\left[{ }^{12} \mathrm{C}\right] /\left[{ }^{13} \mathrm{C}\right]$ (Langer et al. 1984; Tunnard \& Greve 2016).

Furthermore, in intense far-UV environments, selective photodissociation can lead to an increase in $\left[{ }^{12} \mathrm{CO}\right] /\left[{ }^{13} \mathrm{CO}\right]$, since ${ }^{13} \mathrm{CO}$ is readily destroyed given its low abundance, while ${ }^{12} \mathrm{CO}$ will be able to self-shield (Bally \& Langer 1982). However, this effect is expected to play a role only in diffuse gas $\left(A_{\mathrm{V}} \sim 1\right)$ and it is thus doubtful whether selective photodissociation plays a significant role in dusty galaxies, where FUV light undergoes heavy extinction (Casoli et al. 1992; Papadopoulos et al. 2014).

Finally, it is non-trivial to infer ${ }^{12} \mathrm{CO}-$ to- $^{13} \mathrm{CO}$ abundance ratios from their line intensity ratios. This is because the ${ }^{12} \mathrm{CO}$ lines are optically thick, while the rarer ${ }^{13} \mathrm{CO}$ lines tend to be optically thin (e.g., Casoli et al. 1992; Aalto et al. 1995). Optical depth effects can thus complicate the picture, since in the case where ${ }^{12} \mathrm{CO}$ is optically thick the ${ }^{12} \mathrm{CO} /{ }^{13} \mathrm{CO}$ line intensity ratio will be an upper limit to the $\left[{ }^{12} \mathrm{CO}\right] /\left[{ }^{13} \mathrm{CO}\right]$ abundance ratio.

In Fig. 9 (left), we compare our measurements of the ${ }^{12} \mathrm{CO}$ versus ${ }^{13} \mathrm{CO}$ ratio with the literature. We found a mean ratio of $26.1_{-3.5}^{+4.5}$, which is two times higher than the measurements of Spilker et al. (2014) using the stacked spectra of all SPT SMGs observed by ALMA during the cycle 0. Our sample was selected because of their high apparent luminosity and could be biased towards objects with a stronger contribution of the recent star formation to the ISM enrichment. It is therefore not surprising to find a lower ${ }^{13} \mathrm{CO}$ abundance, and thus a higher ${ }^{12} \mathrm{CO} /{ }^{13} \mathrm{CO}$ ratio, in our subsample. Weiß et al. (2013) reported a detection of ${ }^{13} \mathrm{CO}(4-3)$ in SPT0529-54 and a tentative detection in SPT053250 in the initial SPT SMG redshift-search program. The fluxes of these two lines, not published in the original paper, are 1.36 $\pm 0.24 \mathrm{Jy} \mathrm{km} \mathrm{s}^{-1}$ and $1.07 \pm 0.32 \mathrm{Jy} \mathrm{km} \mathrm{s}^{-1}$, respectively, while the ${ }^{12} \mathrm{CO}(4-3)$ fluxes are $8.32 \pm 0.34 \mathrm{Jy} \mathrm{km} \mathrm{s}^{-1}$ and $14.89 \pm$ $0.41 \mathrm{Jy} \mathrm{km} \mathrm{s}^{-1}$. These two sources have much lower ratios than the new objects presented in this paper. This is not surprising, 


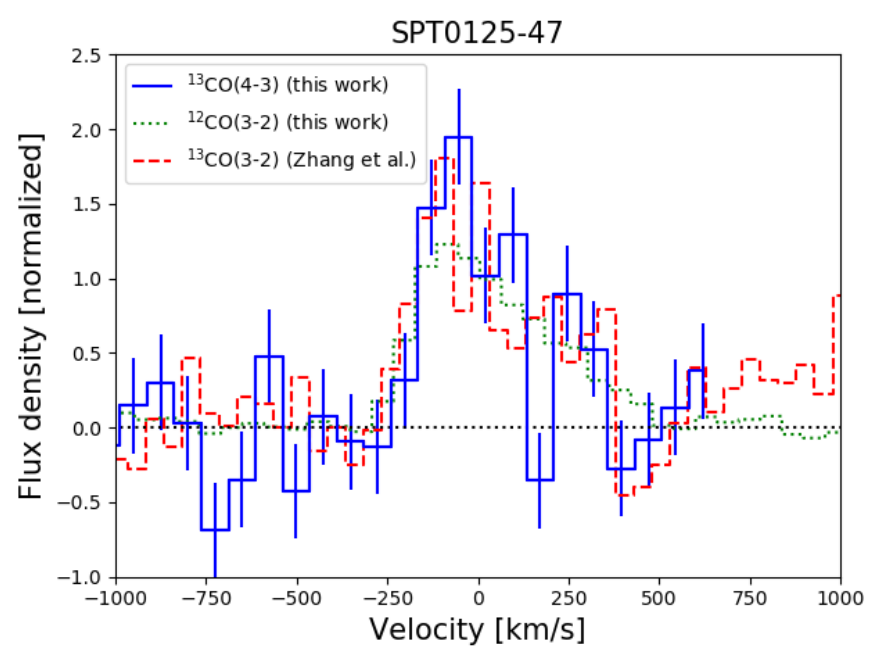

Fig. 10. Comparison of the velocity profiles of the ${ }^{13} \mathrm{CO}(4-3)$ (blue solid line) and ${ }^{12} \mathrm{CO}(3-2)$ lines (green dotted line). The uncertainties on the ${ }^{12} \mathrm{CO}$ spectrum are not plotted, since they are negligible compared with the ${ }^{13} \mathrm{CO}$ ones. We normalized all the lines to have $\int S_{v} \mathrm{~d} v=500 \mathrm{Jy} \mathrm{km} \mathrm{s}^{-1}$. With this normalization, the peak flux of a line with a typical $500 \mathrm{~km} \mathrm{~s}^{-1}$ width is unity. The red dashed line is the ${ }^{13} \mathrm{CO}(3-2)$ profile measured by Zhang et al. (2018).

since these serendipitous detections are biased towards sources with particularly bright ${ }^{13} \mathrm{CO}(4-3)$ lines or with a positive fluctuation of the noise at the position of this line, while the ${ }^{12} \mathrm{CO}$ lines are brighter and thus systematically detected. Consequently, the ${ }^{12} \mathrm{CO} /{ }^{13} \mathrm{CO}$ ratios of these serendipitous detections are biased toward lower values. In Appendix E, we present a simulation illustrating this effect.

Our mean ratio corresponds to the upper envelope of the local sample of Costagliola et al. (2011) in the LIRG regime, but is lower than previous high- $z$ measurements on individual objects of Henkel et al. (2010, Cloverleaf) and Danielson et al. (2013, SMM J2135-0102). Despite our sources having a deficit of ${ }^{13} \mathrm{CO}$ compared to the average values in the local Universe, their isotopic flux ratio is compatible with some local LIRGs. However, it is hard to know if it is really due to similar ${ }^{13} \mathrm{CO}$ abundances in local LIRGs and DSFGs or if the ${ }^{13} \mathrm{CO}$ abundance evolves and is compensated by optical depth effects. The presence of ${ }^{13} \mathrm{CO}$ shows that our objects are clearly not experiencing a first giant starburst fed by pristine gas.

\subsection{Comparison of ${ }^{13} \mathrm{CO}$ and ${ }^{12} \mathrm{CO}$ line profiles in SPT0125-47}

If the various parts of a galaxy have very different ages and ISM enrichment, we might observe very different ${ }^{12} \mathrm{CO}$ and ${ }^{13} \mathrm{CO}$ profiles. In particular, we expect a stronger enrichment of ${ }^{13} \mathrm{CO}$ in the center of galaxies, which harbor older stellar populations responsible for producing this isotope. Furthermore, levels of accretion of pristine cosmological gas onto the galaxy are lower in the central regions, resulting in less dilution of the enriched gas already there. In SPT0125-47, however, we found very similar ${ }^{12} \mathrm{CO}$ and ${ }^{13} \mathrm{CO}$ line profiles, suggesting the abundance ratio of the two molecules remains roughly constant throughout.

In Fig. 10, we compare the velocity profiles of ${ }^{13} \mathrm{CO}(4-3)$ and ${ }^{12} \mathrm{CO}(3-2)$. In addition, we included the ${ }^{13} \mathrm{CO}(3-2)$ measurement of Zhang et al. (2018). Overall, the ${ }^{12} \mathrm{CO}$ and ${ }^{13} \mathrm{CO}$ profiles are rather similar. The two channels at the peak of ${ }^{13} \mathrm{CO}(4-3)$ are $1 \sigma$ and $2 \sigma$ above the ${ }^{12} \mathrm{CO}(3-2)$, but this is not the case for
${ }^{12} \mathrm{CO}(3-2)$. This small tension thus seems to be caused by noise. The similarity of the three profiles suggest that the maturity of the ISM across the different regions of SPT0125-47 is relatively homogeneous. These similar profiles are also a reassuring indication that there should be no strong differential magnification affecting this source.

\section{Conclusion}

In this paper, we presented the main results of our ALMA program targeting dense-gas lines $\left(\mathrm{HCN}(5-4), \mathrm{HCO}^{+}(5-4)\right.$, and $\mathrm{HNC}(5-4)$ ) in five strongly-lensed DSFGs at $2.5<z<4$. We obtained in total two detections $(S / N>5)$ in SPT0125-47 and four tentative detections $(S / N \sim 3)$ of dense-gas lines in other sources. In addition, our observations yielded two detections (one of which is tentative), as well as two upper limits, of ${ }^{13} \mathrm{CO}(4-3)$. We also detected [CI](1-0) with an $\mathrm{S} / \mathrm{N}$ of 36 in SPT0125-47. Finally, we developed a method to derive unbiased average properties of our sample to compare them with the lowand high-redshift literature. From this information, we draw the following conclusions:

- The average $\mathrm{HCN}(5-4)$ luminosity of our sample is formally a factor of $\sim 1.7$ lower than what is expected from the local $L_{\mathrm{HCN}}^{\prime}-L_{\mathrm{IR}}$ linear relation of Zhang et al. (2014). However, if we take into account the sample variance, this tension decreases to $2 \sigma$. Furthermore, we cannot rule out the introduction of systematic uncertainties in the conversion from $\mathrm{HCN}(5-4)$ to $\mathrm{HCN}(4-3)$ when comparing to the Zhang et al. (2014) sample. A similar trend, however, was reported in high- $z$ quasars for high- $J$ transitions by Riechers et al. (2007a) and is also expected by such theoretical models as that of Krumholz \& Thompson (2007) for high-SFR systems.

- Our sample has a mean $I_{\mathrm{HCO}^{+}(5-4)} / I_{\mathrm{HCN} \text { (5-4) flux ratio of }}$ $1.00_{-0.19}^{+0.23}$. This ratio is close to unity and is similar to what is found in local star-forming systems. Based on low-redshift observations, we would have expected a higher ratio for lowmetallicity galaxies or a lower ratio in AGN-dominated systems.

- After deblending the $\mathrm{HNC}(5-4)$ and $\mathrm{CN}(4-3)$ lines, we found an average $I_{\mathrm{HNC}(5-4)} / I_{\mathrm{HCN}(5-4)}$ flux ratio of $1.03_{-0.39}^{+0.59}$, placing our objects at the border between the PDR-dominated regime corresponding to a ratio below unity and the highratio regime caused by $\mathrm{XDR}$ and/or mid-IR pumping of HNC.

- We inferred the $\mathrm{HCN}(5-4) /[\mathrm{CI}](1-0)$ ratio for our sources and found it to be higher than what is measured in LIRGs and ULIRGs in the local Universe. To the extent that this ratio is a proxy of the dense-gas fraction, this finding would suggest that our SPT sources have a larger dense-gas fraction than local (U)LIRGs. In the diagram showing the dense-gas fraction versus the star formation efficiency relative to the bulk gas mass, our sources are located on the same linear relation as the local galaxies.

- In SPT0125-47, we compared the profiles of our $5.5 \sigma$ detections of $\mathrm{HCN}(5-4)$ and $\mathrm{HCO}^{+}(5-4)$, our $36 \sigma$ detection of $[\mathrm{CI}](1-0)$, and the $\mathrm{CO}(4-3)$ line from the original redshiftsearch observation. All these lines have the same asymmetric profile. This suggests that the low- and high-density molecular gas is distributed in the same way across this galaxy. This similarity of the line profiles also suggests the absence of significant differential lensing.

- Concerning the CO isotopologues, our sample has a mean $I^{{ }^{12} \mathrm{CO}(4-3)} / I_{13} \mathrm{CO}(4-3)$ flux ratio of $26.1_{-3.5}^{+4.5}$, which is $30 \%$ 
lower than the previously observed high- $z$ galaxies but $50 \%$ higher than typical local LIRGs. Even if the interpretation of this single line ratio is ambiguous, it tends to indicate that the ISM of our objects has already been enriched by intermediate-mass stars.

Even if the interpretation of these lines is non-trivial, our study allows us to draw a first preliminary portrait of the dense ISM in the SPT SMG sources. Previous works had already established that they had large gas reservoirs (Aravena et al. 2016), but we can now confirm that they have also a high DGF, as traced by $\mathrm{HCN}(5-4) /[\mathrm{CI}](1-0)$. A high DGF naturally leads to a higher star formation efficiency measured relative to the bulk gas mass, that is, a high $L_{\mathrm{IR}} / L_{\mathrm{CO}(1-0)}^{\prime}$ or, as in our case, a high $L_{\mathrm{IR}} / L_{[\mathrm{CI}](1-0)}^{\prime}$. The combination of large reservoirs and high SFEs might be sufficient to explain the prodigious SFRs observed for the SPT sources. The ratios between dense-gas tracers are compatible with PDR-dominated objects and no evidence of AGN activity has been found so far.

Combining our sample with the previously observed highredshift galaxies dominated by star formation, we currently have dense-gas data for less than ten systems. Our analysis revealed some small tensions with other studies. However, their statistical significance is often below $2 \sigma$. We need larger samples and higher $\mathrm{S} / \mathrm{N}$ ratios to identify whether these differences are real and if they are linked to the physical properties of the galaxies. Our study shows that dense-gas tracers can be detected in $\sim 1 \mathrm{~h}$ with ALMA in highly-magnified objects and we can realistically hope to build a sample containing 20-30 objects in the coming years. Unfortunately, $\mathrm{HCN}$ is a bit fainter than expected from the observations at low redshift, but this bad news is totally compensated for by the fact that $\mathrm{HCO}^{+}$and $\mathrm{HNC}$ are at least as bright and can be detected by the same observations.

Acknowledgements. MB would like to thank Zhi-Yu Zhang, Chentao Yang, and Alain Omont for providing their data points and/or the insightful discussions that we had about dense-gas tracers. This paper makes use of the following ALMA data: ADS/JAO.ALMA\#2016.1.00065.S, ADS/JAO.ALMA\#2011.0.00957.S ALMA is a partnership of ESO (representing its member states), NSF (USA), and NINS (Japan), together with NRC (Canada), MOST and ASIAA (Taiwan), and KASI (Republic of Korea), in cooperation with the Republic of Chile. The Joint ALMA Observatory is operated by ESO, AUI/NRAO, and NAOJ. MA acknowledges partial support from FONDECYT through grant 1140099.

\section{References}

Aalto, S., Booth, R. S., Black, J. H., \& Johansson, L. E. B. 1995, A\&A, 300, 369 Aalto, S., Spaans, M., Wiedner, M. C., \& Hüttemeister, S. 2007, A\&A, 464, 193 Aalto, S., Martín, S., Costagliola, F., et al. 2015, A\&A, 584, A42 Aravena, M., Decarli, R., Walter, F., et al. 2016, ApJ, 833, 68 Bally, J., \& Langer, W. D. 1982, ApJ, 255, 143

Barvainis, R., Maloney, P., Antonucci, R., \& Alloin, D. 1997, ApJ, 484, 695 Béthermin, M., Daddi, E., Magdis, G., et al. 2015, A\&A, 573, A113

Bigiel, F., Leroy, A. K., Jiménez-Donaire, M. J., et al. 2016, ApJ, 822, L26 Blain, A. W., Barnard, V. E., \& Chapman, S. C. 2003, MNRAS, 338, 733 Bothwell, M. S., Maiolino, R., Peng, Y., et al. 2016, MNRAS, 455, 1156

Braine, J., Shimajiri, Y., André, P., et al. 2017, A\&A, 597, A44

Bussmann, R. S., Narayanan, D., Shirley, Y. L., et al. 2008, ApJ, 681, L73

Carilli, C. L., \& Walter, F. 2013, ARA\&A, 51, 105

Carilli, C. L., Solomon, P., Vanden Bout, P., et al. 2005, ApJ, 618, 586

Casey, C. M., Narayanan, D., \& Cooray, A. 2014, Phys. Rep., 541, 45

Casoli, F., Dupraz, C., \& Combes, F. 1992, A\&A, 264, 55

Chabrier, G. 2003, PASP, 115, 763

Costagliola, F., Aalto, S., Rodriguez, M. I., et al. 2011, A\&A, 528, A30

Daddi, E., Elbaz, D., Walter, F., et al. 2010, ApJ, 714, L118

Danielson, A. L. R., Swinbank, A. M., Smail, I., et al. 2011, MNRAS, 410, 1687

Danielson, A. L. R., Swinbank, A. M., Smail, I., et al. 2013, MNRAS, 436, 2793

Dessauges-Zavadsky, M., Zamojski, M., Schaerer, D., et al. 2015, A\&A, 577, A50

Engel, H., Tacconi, L. J., Davies, R. I., et al. 2010, ApJ, 724, 233
Faucher-Giguère, C.-A., Quataert, E., \& Hopkins, P. F. 2013, MNRAS, 433, 1970 Fensch, J., Renaud, F., Bournaud, F., et al. 2017, MNRAS, 465, 1934 Galametz, M., Zhang, Z.-Y., Immer, K., et al. 2016, MNRAS, 462, L36 Gao, Y., \& Solomon, P. M. 2004, ApJ, 606, 271

Gao, Y., Carilli, C.L., Solomon, P.M., \& Vanden Bout, P.A. 2007, ApJ, 660, L93 García-Burillo, S., Graciá-Carpio, J., Guélin, M., et al. 2006, ApJ, 645, L17 García-Burillo, S., Usero, A., Alonso-Herrero, A., et al. 2012, A\&A, 539, A8 Genzel, R., Tacconi, L. J., Gracia-Carpio, J., et al. 2010, MNRAS, 407, 2091 Graciá-Carpio, J., García-Burillo, S., Planesas, P., \& Colina, L. 2006, ApJ, 640, L135

Graciá-Carpio, J., García-Burillo, S., \& Planesas, P. 2008, Ap\&SS, 313, 331

Greve, T. R., Hainline, L. J., Blain, A. W., et al. 2006, AJ, 132, 1938

Greve, T. R., Leonidaki, I., Xilouris, E. M., et al. 2014, ApJ, 794, 142

Grudić, M. Y., Hopkins, P. F., Faucher-Giguére, C. A., et al. 2018, MNRAS, 475, 3511

Guélin, M., Salomé, P., Neri, R., et al. 2007, A\&A, 462, L45

Gullberg, B., De Breuck, C., Vieira, J. D., et al. 2015, MNRAS, 449, 2883

Hayward, C. C., Kereš, D., Jonsson, P., et al. 2011, ApJ, 743, 159

Hayward, C. C., Narayanan, D., Kereš, D., et al. 2013, MNRAS, 428, 2529

Heiderman, A., Evans, II., N. J., Allen, L. E., Huard, T., \& Heyer, M. 2010, ApJ, 723, 1019

Henkel, C., Downes, D., Weiß, A., Riechers, D., \& Walter, F. 2010, A\&A, 516, A111

Hezaveh, Y. D., Marrone, D. P., \& Holder, G. P. 2012, ApJ, 761, 20

Hirota, T., Yamamoto, S., Mikami, H., \& Ohishi, M. 1998, ApJ, 503, 717

Hodge, J. A., Riechers, D., Decarli, R., et al. 2015, ApJ, 798, L18

Hopkins, P. F., Narayanan, D., Murray, N., \& Quataert, E. 2013, MNRAS, 433, 69

Hughes, G. L., Gibson, B. K., Carigi, L., et al. 2008, MNRAS, 390, 1710

Imanishi, M., Nakanishi, K., \& Izumi, T. 2016, AJ, 152, 218

Iono, D., Wilson, C. D., Yun, M. S., et al. 2009, ApJ, 695, 1537

Izumi, T., Kohno, K., Aalto, S., et al. 2016, ApJ, 818, 42

Juneau, S., Narayanan, D. T., Moustakas, J., et al. 2009, ApJ, 707, 1217

Kamenetzky, J., Rangwala, N., Glenn, J., Maloney, P. R., \& Conley, A. 2016, ApJ, 829, 93

Kauffmann, J., Goldsmith, P. F., Melnick, G., et al. 2017, A\&A, 605, L5

Keating, G. K., Marrone, D. P., Bower, G. C., et al. 2016, ApJ, 830, 34

Knudsen, K. K., Walter, F., Weiss, A., et al. 2007, ApJ, 666, 156

Krumholz, M. R., \& Thompson, T. A. 2007, ApJ, 669, 289

Lada, C. J., Lombardi, M., \& Alves, J. F. 2010, ApJ, 724, 687

Lada, C. J., Forbrich, J., Lombardi, M., \& Alves, J. F. 2012, ApJ, 745, 190

Langer, W. D., Graedel, T. E., Frerking, M. A., \& Armentrout, P. B. 1984, ApJ, 277,581

Larson, R. B. 1994, in The Structure and Content of Molecular Clouds, eds. T. L. Wilson, \& K. J. Johnston (Berlin: Springer Verlag), Lect. Notes Phys., 439, 13

Liu, D., Gao, Y., Isaak, K., et al. 2015, ApJ, 810, L14

Magdis, G. E., Elbaz, D., Dickinson, M., et al. 2011, A\&A, 534, A15

Magdis, G. E., Daddi, E., Béthermin, M., et al. 2012, ApJ, 760, 6

Mangum, J. G., Darling, J., Menten, K. M., \& Henkel, C. 2008, ApJ, 673, 832

Mangum, J. G., Darling, J., Henkel, C., \& Menten, K. M. 2013, ApJ, 766, 108

McMullin, J. P., Waters, B., Schiebel, D., Young, W., \& Golap, K. 2007, ASPC, 376,127

Mills, E. A. C., Güsten, R., Requena-Torres, M. A., \& Morris, M. R. 2013, ApJ, 779,47

Narayanan, D., Groppi, C. E., Kulesa, C. A., \& Walker, C. K. 2005, ApJ, 630, 269

Narayanan, D., Cox, T. J., \& Hernquist, L. 2008a, ApJ, 681, L77

Narayanan, D., Cox, T. J., Shirley, Y., et al. 2008b, ApJ, 684, 996

Narayanan, D., Cox, T. J., Hayward, C. C., \& Hernquist, L. 2011, MNRAS, 412, 287

Narayanan, D., Turk, M., Feldmann, R., et al. 2015, Nature, 525, 496

Onus, A., Krumholz, M.R., \& Federrath, C. 2018, MNRAS, 479, 1702

Oteo, I., Zwaan, M. A., Ivison, R. J., Smail, I., \& Biggs, A. D. 2016, ApJ, 822, 36

Oteo, I., Zhang, Z., Yang, C., et al. 2017, ApJ, 850, 170

Papadopoulos, P. P. 2007, ApJ, 656, 792

Papadopoulos, P. P. 2010, ApJ, 720, 226

Papadopoulos, P. P., Thi, W.-F., Miniati, F., \& Viti, S. 2011, MNRAS, 414, 1705

Papadopoulos, P. P., Zhang, Z.-Y., Xilouris, E. M., et al. 2014, ApJ, 788, 153

Planck Collaboration XIII. 2016, A\&A, 594, A13

Popping, G., Pérez-Beaupuits, J. P., Spaans, M., Trager, S. C., \& Somerville, R. S. 2014, MNRAS, 444, 1301

Renaud, F., Bournaud, F., Kraljic, K., \& Duc, P.-A. 2014, MNRAS, 442, L33

Riechers, D. A., Walter, F., Carilli, C. L., \& Bertoldi, F. 2007a, ApJ, 671, L13

Riechers, D. A., Walter, F., Cox, P., et al. 2007b, ApJ, 666, 778

Riechers, D. A., Weiß, A., Walter, F., \& Wagg, J. 2010, ApJ, 725, 1032 
Romano, D., Matteucci, F., Zhang, Z.-Y., Papadopoulos, P. P., \& Ivison, R. J. 2017, MNRAS, 470, 401

Rosenberg, M. J. F., van der Werf, P. P., Aalto, S., et al. 2015, ApJ, 801, 72

Saintonge, A., Lutz, D., Genzel, R., et al. 2013, ApJ, 778, 2

Sanders, D. B., Mazzarella, J. M., Kim, D.-C., Surace, J. A., \& Soifer, B. T. 2003, AJ, 126, 1607

Sargent, M. T., Daddi, E., Béthermin, M., et al. 2014, ApJ, 793, 19

Schilke, P., Walmsley, C. M., Pineau Des Forets, G., et al. 1992, A\&A, 256, 595

Schinnerer, E., Groves, B., Sargent, M. T., et al. 2016, ApJ, 833, 112

Scoville, N., Aussel, H., Sheth, K., et al. 2014, ApJ, 783, 84

Scoville, N., Sheth, K., Aussel, H., et al. 2016, ApJ, 820, 83

Serjeant, S. 2012, MNRAS, 424, 2429

Shirley, Y. L. 2015, PASP, 127, 299

Silverman, J. D., Daddi, E., Rodighiero, G., et al. 2015, ApJ, 812, L23

Solomon, P., Vanden Bout, P., Carilli, C., \& Guelin, M. 2003, Nature, 426, 636

Solomon, P.M., \& Vanden Bout, P.A. 2005, ARA\&A, 43, 677

Spilker, J. S., Marrone, D. P., Aguirre, J. E., et al. 2014, ApJ, 785, 149

Spilker, J. S., Marrone, D. P., Aravena, M., et al. 2016, ApJ, 826, 112

Stark, A. A., Bally, J., Wilson, R. W., \& Pound, M. W. 1989, in The Center of the Galaxy, ed. M. Morris, IAU Symp., 136, 129

Strandet, M. L., Weiss, A., Vieira, J. D., et al. 2016, ApJ, 822, 80

Tacconi, L. J., Genzel, R., Neri, R., et al. 2010, Nature, 463, 781
Tacconi, L. J., Neri, R., Genzel, R., et al. 2013, ApJ, 768, 74 Tan, Q., Daddi, E., Magdis, G., et al. 2014, A\&A, 569, A98 Tunnard, R., \& Greve, T. R. 2016, ApJ, 819, 161 Vanden Bout, P.A., Solomon, P.M., \& Maddalena, R.J. 2004, ApJ, 614, L97

Vieira, J. D., Crawford, T. M., Switzer, E. R., et al. 2010, ApJ, 719, 763

Vieira, J. D., Marrone, D. P., Chapman, S. C., et al. 2013, Nature, 495, 344 Wagg, J., Wilner, D. J., Neri, R., Downes, D., \& Wiklind, T. 2005, ApJ, 634, L13

Wang, J., Zhang, Z., \& Shi, Y. 2011, MNRAS, 416, L21

Watson, W. D., Anicich, V. G., \& Huntress, Jr., W. T. 1976, ApJ, 205, L165

Weiß, A., Downes, D., Neri, R., et al. 2007, A\&A, 467, 955

Weiß, A., De Breuck, C., Marrone, D. P., et al. 2013, ApJ, 767, 88

Wilson, T. L., \& Rood, R. 1994, ARA\&A, 32, 191

Wu, J., Evans, II., N. J., Gao, Y., et al. 2005, ApJ, 635, L173

Wu, J., Evans, N., Shirley, Y., \& Knez, C. 2010, ApJS, 188, 313

Yang, C. 2017, PhD Thesis, Institut d'Astrophysique Spatiale, CNRS, Univ. Paris-Sud, Université Paris-Saclay, France; Purple Mountain Observatory, Chinese Academy of Sciences, PR China

Yang, C., Omont, A., Beelen, A., et al. 2017, A\&A, 608, A144

Yao, L., Seaquist, E. R., Kuno, N., \& Dunne, L. 2003, ApJ, 588, 771

Zhang, Z.-Y., Gao, Y., Henkel, C., et al. 2014, ApJ, 784, L31

Zhang, Z.-Y., Romano, D., Ivison, R. J., Papadopoulos, P. P., \& Matteucci, F. 2018, Nature, 558, 260 


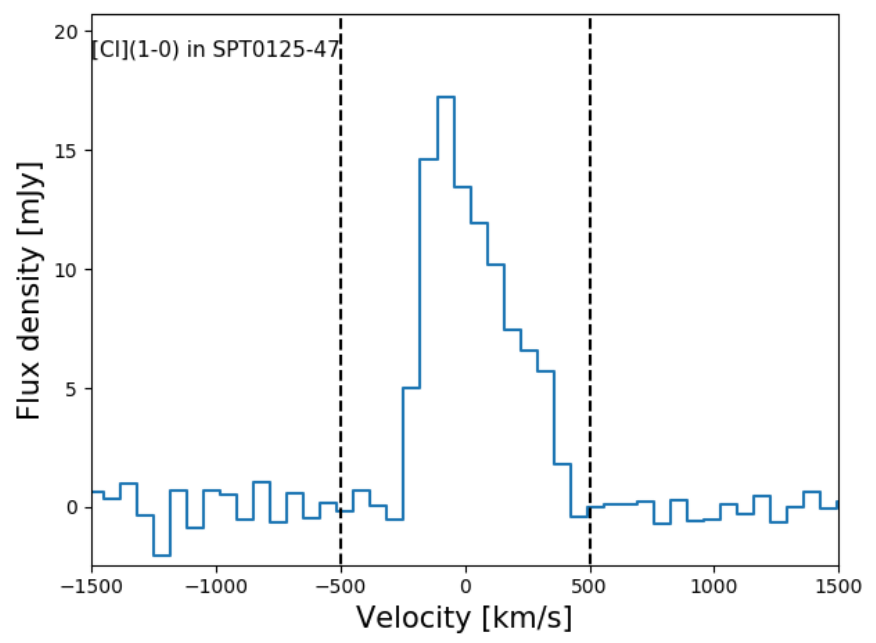

Fig. A.1. Detection of [CI](1-0) in SPT0125-47. The continuum has been subtracted from the spectrum. The vertical dashed lines indicate the window in which the spectrum was integrated to derive the line flux presented in Table 3.

\section{Appendix A: High-S/N detection of $[\mathrm{Cl}](1-0)$ in SPT0125-47}

We detected the [CI](1-0) line of SPT0125-47 for first time. This relatively bright line was not covered by the original band-3 spectral scan used to determine the redshift of the source, but is located in the other side band of our new, deep ALMA band-4 observations of dense-gas lines. We produced a spectrum using the same method as in Sects. 2.2 and 2.3. The line is detected at a very high $\mathrm{S} / \mathrm{N}$ and exhibits an asymmetric profile (see Fig. A.1), making a Gaussian model not appropriate. We measured the flux in a window set manually and covering the full line emission. We derived the uncertainties using the standard deviation outside the frequency window, where the line is located. The line is detected with an $S / N$ of 36 . Table 3 contains the $[\mathrm{CI}](1-0)$ flux of SPT0125-47.

\section{Appendix B: Checking the consistency between our line-extraction method and the classical moment-zero map approach}

Our extraction method presented in Sect. 2.3 relies on the quality of the Gaussian assumption for the line profile. The [CI](1-0) profile shown in Appendix A has a very high $\mathrm{S} / \mathrm{N}$ and is clearly non-Gaussian. Usually, the best way to deal with non-Gaussian lines is to produce a moment-zero map by summing all the channels, which contains flux from the lines. However, this method works only if the continuum has been previously subtracted in the uv plane (uvcontsub in CASA).

Unfortunately, this subtraction is difficult in the case of our data, since we can only use a couple of clean continuum channels between $\mathrm{HCO}^{+}(5-4)$ and $\mathrm{HNC}(5-4)$ in the side band of the dense-gas tracers. This is caused by the presence of several lines in this side band, which can be particularly broad, especially in SPT0551-50. The line-versus-continuum flux density ratio is of the order of unity and the slope of the continuum versus frequency inside a spectral window is non-negligible. This makes this task even more difficult. We performed various tests and realized that the continuum subtraction was very sensitive to our parameter choices, creating important systematics. For instance, with SPT0551-50, the recovered flux in the moment-zero map varies by more than $1 \sigma$ if we remove a flat background measured between $\mathrm{HCO}^{+}(5-4)$ and $\mathrm{HNC}(5-4)$ or if we use a firstorder baseline using in addition the other side band to constrain the slope. This is even worse for the three sources at low $\mathrm{S} / \mathrm{N}$ (SPT0103-45, SPT0125-50, SPT0300-46) for which the flux of the line can vary by a factor of two depending on how the continuum was subtracted.

These problems motivated our choice to use our own method introduced in Sect. 2.3 to derive line fluxes. However, we performed a comparison between our approach and the momentzero measurements using a first-order continuum subtraction. We restrict this comparison to SPT0125-47 and SPT0551-50 for which we can clearly identify the channels contaminated by the lines and thus reliably subtract the continuum. The results are summarized in Table B.1. The values derived by the two methods are compatible at $1 \sigma$ for all the lines. The uncertainties derived using our own method are slightly higher than the ones obtained with the moment maps. This is expected, since it takes into account the impact of the uncertainties on the baseline level and on the line width, while the moment-zero method assumes implicitly that the continuum is subtracted perfectly and uses a fixed velocity window to integrate the line flux. While they are slightly higher, the uncertainties provided by our fitting method are thus probably more realistic. Concerning the $\mathrm{S} / \mathrm{N}$ ratios, the estimates based on the moment-zero maps are close to the ones derived using the ratio between the peak line flux density determined with our method and the uncertainty on it $\left(S_{\text {peak }} / \sigma_{S_{\text {peak }}}\right)$.

Table B.1. Comparison between our measurements preformed using the method described in Sect. 2.3 and the classical approach based on the moment-zero map (Appendix B).

\begin{tabular}{|c|c|c|c|c|c|}
\hline \multirow[t]{2}{*}{ Source } & \multirow[t]{2}{*}{ Line } & \multicolumn{2}{|c|}{ Flux density $\left(\mathrm{Jy} \mathrm{km} \mathrm{s}^{-1}\right)$} & \multicolumn{2}{|c|}{$S / N$} \\
\hline & & Fit + bootstrap & Moment-zero & Fit + bootstrap $^{a}$ & Moment-zero \\
\hline \multirow[t]{4}{*}{ SPT0125-47 } & ${ }^{13} \mathrm{CO}(4-3)$ & $1.15 \pm 0.22$ & $0.90 \pm 0.15$ & 7.2 & 6.0 \\
\hline & $\operatorname{HCN}(5-4)$ & $0.96 \pm 0.23$ & $0.91 \pm 0.15$ & 5.3 & 6.0 \\
\hline & $\mathrm{HCO}^{+}(5-4)$ & $1.01 \pm 0.20$ & $0.91 \pm 0.14$ & 5.6 & 6.5 \\
\hline & {$[\mathrm{CI}](1-0)$} & $6.36 \pm 0.27$ & $6.21 \pm 0.13$ & 27 & 41 \\
\hline \multirow[t]{4}{*}{ SPT0551-50 } & ${ }^{13} \mathrm{CO}(4-3)$ & $0.32 \pm 0.15$ & $0.37 \pm 0.07$ & 3.4 & 5.3 \\
\hline & $\mathrm{HCN}(5-4)$ & $0.22 \pm 0.11$ & $0.25 \pm 0.08$ & 3.0 & 3.2 \\
\hline & $\mathrm{HCO}^{+}(5-4)$ & $0.17 \pm 0.07$ & $0.24 \pm 0.07$ & 3.1 & 3.4 \\
\hline & $\mathrm{HNC}+\mathrm{CN}$ & $0.25 \pm 0.09$ & $0.26 \pm 0.08$ & 2.6 & 3.3 \\
\hline
\end{tabular}

Notes. ${ }^{(a)}$ The $\mathrm{S} / \mathrm{N}$ of our fitting method is determined by computing $S_{\text {peak }} / \sigma_{S_{\text {peak }}}$. 
In SPT0125-47, the S/N and the line flux of [CI](1-0) based on the method presented in Appendix A is between the momentzero and the fitting method and we will keep this intermediate value for analysis. Indeed, since the $[\mathrm{CI}]$ flux is used to compute ratios with much more uncertain quantities, these small differences do not have any significant impact on our results. Our Gaussian fit and the moment-zero method agree at the 3\% level. This confirms that the Gaussian approximation is a reasonable assumption for lines detected at $\sim 5 \sigma$. In this case, the systematic effect induced by the non-Gaussian profile of $[\mathrm{CI}]$ is only $0.15 \sigma$.

\section{Appendix C: Validation of our method deriving mean luminosities and ratios}

In Sects. 2.5 and 2.6, we presented a statistical approach to derive mean luminosities and line ratios without being biased towards detections. In this appendix, we present the simulation used to validate our method.

We generated 500 simulated spectra at $z \sim 3.2$ of two spectral windows covering ${ }^{13} \mathrm{CO}(4-3), \mathrm{HCN}(5-4), \mathrm{HCO}^{+}(5-4)$, HNC(5-4), and CN(4-3) (104.6-108.35 GHz). This redshift was chosen because it allows us to cover all the line at the same time and it is close to the mean redshift of the real sample. With these 500 spectra in hand, we reduced the statistical uncertainties on the derived mean quantities by a factor of ten compared to our real sample. This allows us to check if small systematic biases are affecting our approach.

For each source, we drew the fluxes of the ${ }^{13} \mathrm{CO}(4-3)$, $\mathrm{HCN}(5-4), \mathrm{HCO}^{+}(5-4)$, and $\mathrm{HNC}(5-4)$ lines from an uniform distribution between $0 \mathrm{Jy} \mathrm{km} \mathrm{s}^{-1}$ and $0.3 \mathrm{Jy} \mathrm{km} \mathrm{s}^{-1}$, which is the typical range in our real sample if we leave out the exceptionally bright SPT0125-47. We used a maximum flux of $0.187 \mathrm{Jy} \mathrm{km} \mathrm{s}^{-1}$ for $\mathrm{CN}(4-3)$ to be consistent with the mean observed flux ratio with HNC(5-4) (1.6, see Sect. 2.6). For all the lines of a given source, we used the same line width drawn from a uniform distribution centered on $370 \mathrm{~km} \mathrm{~s}^{-1}$ and with a half width of $130 \mathrm{~km} \mathrm{~s}^{-1}$ as measured by Aravena et al. (2016) for low- $J$ CO lines in the SPT SMG sample. Finally, we added a random Gaussian noise of $0.2 \mathrm{mJy}$ per channel of $31.25 \mathrm{GHz}$ (resolution of SPT0125-47 after rebinning). We extracted the lines in these simulated spectra using the method presented in Sect. 2.3. For HNC(5-4) and $\mathrm{CN}(4-3)$, following Sect. 2.6, we performed a second run assuming a positivity prior and no velocity offset to obtain a better deblending. The median $\mathrm{S} / \mathrm{N}$ of the ${ }^{13} \mathrm{CO}(4-3)$, $\mathrm{HCN}(5-4)$, and $\mathrm{HCO}^{+}(5-4)$ lines is $2.9,2.8$, and 3.1, respectively. Roughly half of our simulated sample is thus not even tentatively detected.

In Table C.1, we compare the true fluxes injected into our simulation with the measured ones. The maximal difference between these two quantities is $6.3 \%$. This demonstrates that our method is reasonably accurate to derive the mean flux of a sample even if a large fraction of the objects is not detected. This difference of $6.3 \%$ obtained for ${ }^{13} \mathrm{CO}(4-3)$ is significant at $4 \sigma$, when we consider the uncertainty corresponding to the instrumental noise in our simulated spectra. There are thus residual systematic effects, but they remain small compared to the typical uncertainties on our measurements based on four or five sources $(\gtrsim 20 \%)$. In addition, we found that the measured fluxes of $>3 \sigma$ (tentative) detections are overestimated on average by $28 \%, 41 \%$, and $13 \%$ for ${ }^{13} \mathrm{CO}(4-3), \mathrm{HCN}(5-4)$, and $\mathrm{HCO}^{+}(5-4)$, respectively. Our statistical approach is thus clearly better than computing naively the mean flux of the detected sources.
Table C.1. Results of our simulation validating our method to derive mean luminosities and ratios (see Appendix C).

\begin{tabular}{lccccc}
\hline \hline Observable & $\left\langle X_{\text {true }}\right\rangle$ & $\left\langle X_{\text {mes }}\right\rangle$ & $\frac{\left\langle X_{\text {mes }}\right\rangle}{\left\langle X_{\text {ture }}\right\rangle}$ & $\frac{\sigma_{\text {full }}\left(\left\langle X_{\text {mes }}\right\rangle\right)}{\left\langle X_{\text {true }}\right\rangle}$ & $\frac{\sigma_{5}\left(\left\langle X_{\text {mes }}\right\rangle\right)}{\left\langle X_{\text {true }}\right\rangle}$ \\
\hline \multicolumn{7}{c}{ Line fluxes in Jy km s } \\
\end{tabular}

Notes. For line fluxes, the true sample mean value $\left\langle X_{\text {true }}\right\rangle$ is the mean of the 500 injected fluxes of our simulated sample. As justified in Sect. 2.5, the mean line flux ratios are derived by dividing the mean flux of a given line by the mean flux of another line. The fluxes measured using our line extraction tool are used to derive $\left\langle X_{\text {mes }}\right\rangle \cdot \frac{\left\langle X_{\text {mes }}\right\rangle}{\left\langle X_{\text {true }}\right\rangle}$ must be close to unity if the method is not biased. $\frac{\sigma_{\text {full }}\left(\left\langle X_{\text {mes }}\right\rangle\right)}{\left\langle X_{\text {true }}\right\rangle}$ is the uncertainty on this ratio caused by the instrumental noise. Finally, by drawing 1000 subsamples of five sources from our 500 objects without withdrawal, we estimated the mean uncertainty on the measured-versus-true ratio for a subsample of five objects $\frac{\sigma_{5}\left(\left\langle X_{\text {mes }}\right\rangle\right)}{\left\langle X_{\text {true }}\right\rangle}$ as is the case for our real observations.

Finally, we compared the flux ratio derived using our statistical method and the true ratio (Table C.1). We found no significant deviation. The small systematic effects affecting mean line fluxes could compensate for each other. The ratios including blended lines are also well recovered. This is a good demonstration of the accuracy of our approach including for blended lines (Sect. 2.6).

\section{Appendix D: Confirming the correlation between dense-gas fraction and star formation efficiency}

In Fig. D.1, we present a version of Fig. 7 using $\mathrm{CO}(4-3)$ instead of $[\mathrm{CI}](1-0)$. Similarly to the original figure, our results are compatible with a linear relation between DGF and SFE. $\mathrm{CO}(4-3)$ is not a very good tracer of the total gas mass, since it is biased towards denser environments compared to $\mathrm{CO}(1-$ 0 ) and it is affected by the variations of the SLED. However, it is reassuring to find a similar result with this independent tracer.

In Sect. 4.2 and Fig. 7, we discussed the link between the star formation efficiency and the dense-gas fraction using a diagram of the form $\mathrm{A} / \mathrm{C}$ versus $\mathrm{B} / \mathrm{C}$. In this case, $\mathrm{C}$ is a quantity tracing the total molecular gas reservoir of the galaxy. Such diagrams can produce artificial correlations, if the uncertainties on $\mathrm{C}$ are of the order of magnitude of $\mathrm{A} / \mathrm{C}$ or $\mathrm{B} / \mathrm{C}$. This is not expected in our diagram, where we probe more than one order of magnitude on both axes, while the measurements uncertainties on the $[\mathrm{CI}](1-0)$ and $\mathrm{CO}(4-3)$ fluxes are lower than $30 \%$. We tested whether the trend of a DGF increasing with higher SFE remains when we use two different tracers of the total cold gas on each axis (Fig. D.2). There is a slightly larger scatter than in the previous plots, but the trend does not disappear. This larger scatter could come from the addition of the systematic effects affecting each tracer in a different way. 


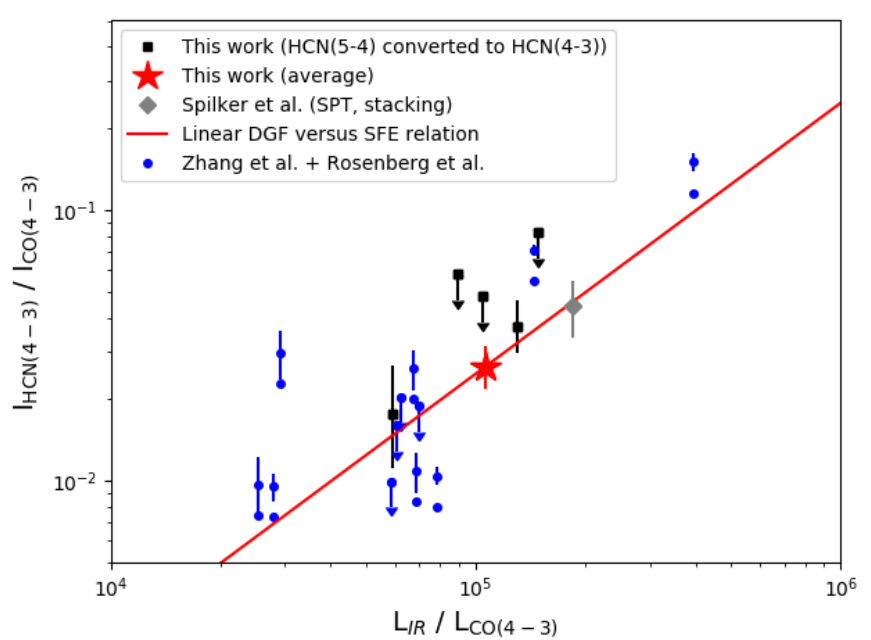

Fig. D.1. Ratio between $\mathrm{HCN}(4-3)$ and $\mathrm{CO}(4-3)$, tracing the dense-gas fraction, as a function of the ratio between the infrared and the $\mathrm{CO}(4-3)$ luminosity, tracing the star formation efficiency. The red solid line is a linear relation between the dense-gas fraction and the star formation efficiency (DGF $\propto$ SFE), which normalization has been set to match the mean value of our sample.
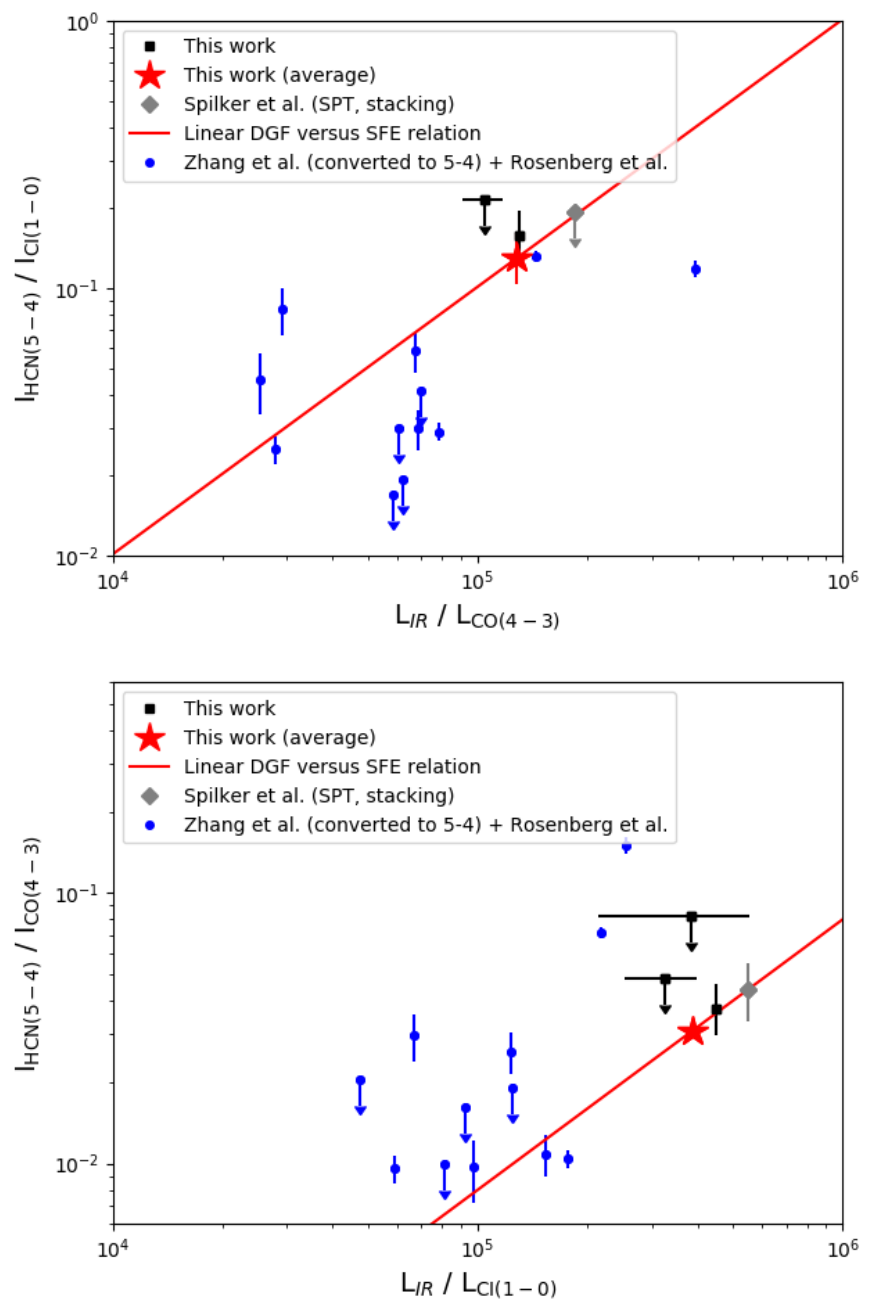

Fig. D.2. Upper panel: dense-gas fraction as a function of star formation efficiency. The data are the same as in Fig. 7, except that we used [CI](10 ) instead of $\mathrm{CO}(4-3)$ on the $y$-axis. Lower panel: same thing as upper panel, except that we switched $\mathrm{CO}(4-3)$ and $[\mathrm{CI}](1-0)$ on the $x$ and $y$ axis.

\section{Appendix E: Biased ${ }^{13} \mathrm{CO} /{ }^{12} \mathrm{CO}$ ratios in samples of serendipitous ${ }^{13} \mathrm{CO}$ detections}

In Sect. 5, we found that serendipitous ${ }^{13} \mathrm{CO}$ detections in the SPT SMG redshift-search program of Weiß et al. (2013) have much lower ${ }^{12} \mathrm{CO} /{ }^{13} \mathrm{CO}$ ratios than both our detections and the stacking of Spilker et al. (2014). To understand this effect, we performed a simplified simulation.

We started from the measured fluxes of the $29{ }^{12} \mathrm{CO}$ lines detected in band 3 in the current SPT SMG sample. These lines are so bright that they are always detected at high $\mathrm{S} / \mathrm{N}$ and we can thus neglect their flux measurement uncertainties. We then generated a "true" ${ }^{13} \mathrm{CO}$ flux by dividing the ${ }^{12} \mathrm{CO}$ by a factor of 26 (the mean line flux ratio found for our sample) and applying a log-normal scatter of $0.3 \mathrm{dex}$, which is approximately what is seen in Fig. 10. We also took into account the impact of the instrumental noise on the measurements of the ${ }^{13} \mathrm{CO}$ fluxes. The noise can vary from one source to another. For simplicity, we assumed the same Gaussian noise $\sigma_{\text {noise }}$ of $0.365 \mathrm{Jy} \mathrm{km} \mathrm{s}^{-1}$ for all the sources, which is the mean of the noise of the two Weiß et al. (2013) detections. We consider as detected only the objects with a "measured" flux larger than $3 \sigma_{\text {noise. Since the }}$ number of these detections can vary significantly from one realization of the intrinsic scatter and the instrumental noise to the other, we repeated this operation 100000 times to minimize the statistical fluctuations. We found on average 3.3 detections with a standard deviation of 1.6. This is compatible with the two detections in the real sample of Weiß et al. (2013).

By construction, the mean ${ }^{12} \mathrm{CO} /{ }^{13} \mathrm{CO}$ ratio of our complete simulated catalog is 26 . In contrast, the mean "true" ratio of the detections is 11.3 with standard deviation of 7.6. The subsample detected in ${ }^{13} \mathrm{CO}$ is thus strongly biased towards low ratios. The mean ratio is even smaller when we compute it using the "measured" ${ }^{13} \mathrm{CO}$ flux: 8.1 with scatter of 4.5 . The instrumental noise thus increases the bias, since the ${ }^{13} \mathrm{CO}$ detections are affected by a severe flux boosting. We found a mean ratio of 1.5 between the "measured" and the "true" flux. These values of the measured ratio are very close to the two serendipitous detections of Weiß et al. (2013).

This simulation demonstrates that the rare serendipitous detections extracted from large samples can be highly biased and should be used with caution. It also shows why it is so important to derive average properties of samples considering also the nondetections to interpret results from a sample (see Sect. 2.5). 